NBER WORKING PAPER SERIES

\title{
INSTITUTIONAL INVESTORS AND INFORMATION ACQUISITION: IMPLICATIONS FOR ASSET PRICES AND INFORMATIONAL EFFICIENCY
}

\author{
Matthijs Breugem \\ Adrian Buss \\ Working Paper 23561 \\ http://www.nber.org/papers/w23561 \\ NATIONAL BUREAU OF ECONOMIC RESEARCH \\ 1050 Massachusetts Avenue \\ Cambridge, MA 02138 \\ June 2017
}

For useful comments and suggestions to this paper and its previous versions, we thank Andrea Buffa (discussant), Will Cong (discussant), Bernard Dumas, Marcin Kacperczyk, Ron Kaniel (discussant), Stijn van Nieuwerburgh, Anna Pavlova (discussant), Joel Peress, Zacharias Sautner, Larissa Schaefer, Guenter Strobl, Raman Uppal, Dimitri Vayanos, Laura Veldkamp, Grigory Vilkov, Jing Zeng and seminar participants at Frankfurt School of Finance and Management, INSEAD, the 2016 European Summer Symposium in Financial Markets, the 2017 Australasian Finance \& Banking Conference, the 2017 Annual Meeting of the American Finance Association, the 2017 Adam Smith Workshops in Asset Pricing and Corporate Finance, the Geneva School of Economics and Management, the CEPR Second Annual Spring Symposium in Financial Economics and the 2017 NBER conference on New Developments in Long-Term Asset Management Conference. This research benefited from the support of the Europlace Institute of Finance and the Labex Louis Bachelier. The views expressed herein are those of the authors and do not necessarily reflect the views of the National Bureau of Economic Research.

NBER working papers are circulated for discussion and comment purposes. They have not been peer-reviewed or been subject to the review by the NBER Board of Directors that accompanies official NBER publications.

(C) 2017 by Matthijs Breugem and Adrian Buss. All rights reserved. Short sections of text, not to exceed two paragraphs, may be quoted without explicit permission provided that full credit, including $\odot$ notice, is given to the source. 
Institutional Investors and Information Acquisition: Implications for Asset Prices and Informational Efficiency Matthijs Breugem and Adrian Buss

NBER Working Paper No. 23561

June 2017

JEL No. G11,G14,G23

\begin{abstract}
We jointly model the information choice and portfolio allocation problem of institutional investors who are concerned about their performance relative to a benchmark. Benchmarking increases an investor's effective risk-aversion, which reduces his willingness to speculate and, consequently, his desire to acquire information. In equilibrium, an increase in the fraction of benchmarked institutional investors leads to a decline in price informativeness, which can cause a decline in the prices of all risky assets and the market portfolio. The decline in price informativeness also leads to a substantial increase in return volatilities and allows nonbenchmarked investors to substantially outperformed benchmarked investors.
\end{abstract}

Matthijs Breugem

Frankfurt School of Finance and Management

Sonnemannstrasse 9-11

60314 Frankfurt

m.breugem@fs.de

Adrian Buss

INSEAD

Boulevard de Constance

77305 Fontainebleau Cedex

FRANCE

adrian.buss@insead.edu 
Over the last decades, the importance of institutional investors in financial markets has grown steadily. For example, the fraction of U.S. equity owned by institutional investors has risen from about 7\% in 1950 to $67 \%$ in 2010 (French (2008), U.S. Securities and Exchange Commission (2013), Stambaugh (2014)), and institutional investors nowadays account for a majority of the transactions and trading volume (Griffin, Harris, and Topaloglu (2003)). Moreover, recently there has been a substantial shift toward benchmarked institutional investors, that is, institutional investors whose performance is evaluated relative to an "index," or benchmark portfolio.

While there is now a growing body of literature studying the asset pricing implications of benchmarking, or, formally, linear performance fees, this literature has concentrated on the case of symmetrically informed investors. ${ }^{1}$ In contrast, the focus of this paper is on the interaction between benchmarking and endogenous information choice. Our objective is to demonstrate how the growth of assets under management by benchmarked institutions affects informational efficiency and asset prices in equilibrium. This will also allow us to emphasize how non-benchmarked investors' portfolio and information choice as well as their performance is influenced by the size of benchmarked institutions.

For this purpose, we develop an equilibrium model with two classes of heterogeneous institutional investors, endogenous information allocation, CRRA-preferences and multiple risky assets to learn about. The model has two key features. First, a fraction of the institutional investors - the benchmarked investors - care not only about their own performance, but also about their performance relative to an index, or, formally, their marginal utility is increasing and their utility is decreasing in the benchmark. This might be due to explicit reasons, for example, performance fees or a fund's "style," or implicit incentives, for instance, through the performance-flow relation. Second, we allow for the joint determination of the institutional investors' portfolio allocation and information choice. That is, the two groups of institutions (benchmarked and non-benchmarked) optimally decide how much information to acquire. They can also extract information from the equilibrium stock prices

\footnotetext{
${ }^{1}$ See, for example, Brennan (1993), Cuoco and Kaniel (2011), Basak and Pavlova (2013), Buffa, Vayanos, and Woolley (2014), Basak and Pavlova (2016), and Buffa and Hodor (2017).
} 
that imperfectly reveal the other investors' information. Otherwise, the framework is kept as simple as possible to illustrate the implications of benchmarking, but also to provide the economic mechanisms that generate them in the clearest possible way.

In the first step, we highlight a novel economic channel through which relative performance concerns affect an investor's investment and information choice. That is, benchmarking leads to an increase in an investor's effective risk-aversion, particular so, as his "surplus performance" relative to the index declines.

We then show, using a closed-form, but approximate, solution that the optimal portfolio shares of a benchmarked institutional investor can be decomposed into three components. The first component is the standard mean-variance portfolio of a non-benchmarked investor. The second component is a hedging portfolio that implies a positive hedging demand for "index stocks" that are included in the benchmark portfolio. The size of this hedging portfolio is increasing in the degree of benchmarking, but is information-insensitive. The third component captures the change in the investor's risk attitude resulting from relative performance concerns and, in general, reduces his demand for risky assets, increasingly so as benchmarking strengthens.

As a direct consequence of the hedging demand for index stocks, a benchmarked institutional investor, on average, over-weights index stocks in his portfolio and under-weights non-index stocks. For typical calibrations, this hedging demand more than offsets the reduction in demand resulting from the lower, effective risk tolerance. Accordingly, benchmarking leads, on average, to an excess demand for index stocks. Most important for our analysis, however, is that in the presence of benchmarking, the investor makes only smaller bets based on private information, or, formally, the sensitivity of his optimal portfolio composition to private information declines. For example, he less aggressively acquires shares of a stock following good news, and vice versa for bad news. This is due to the higher effective risk-aversion and the fact that the hedging demand is information-insensitive.

These changes in a benchmarked investor's portfolio decisions have a direct impact on his information choice. Intuitively, because his trading is less sensitive to private in- 
formation, the marginal benefit from receiving precise private information declines; that is, benchmarked investors value private information less. Hence, as his degree of benchmarking increases, an institutional investor acquires less precise private information.

We next investigate how the growth of assets under management by benchmarked institutions influences informational efficiency and asset prices in equilibrium. For this, we fix the degree of relative performance concerns for the benchmarked investors and, instead, vary their size relative to the overall economy.

We document that, as the fraction of benchmarked institutional investors increases, price informativeness drops - for the index and the non-index stocks. Intuitively, an increase in the share of benchmarked investors implies a shift toward a group of investors who trade less aggressively and also acquire less information. Consequently, aggregate information acquisition declines and less information can be revealed through prices.

Less informative prices make investments into the stocks riskier, because there is more uncertainty about their fundamentals, so that risk-averse investors command a lower price. Hence, as benchmarked investors gain importance, the price of the non-index stocks declines. Due to the positive hedging demand for index stocks, their prices will always be higher than those of comparable non-index stocks, but might still decline in the size of the benchmarked institutions. This is the case if the negative price effect resulting from less informative prices dominates the excess demand from hedging.

The decline in price informativeness also naturally translates into a higher return volatility for all stocks in the economy. For the non-index stocks, the higher return volatility is more than offset by an increase in expected return, so that the Sharpe ratio increases as the fraction of benchmarked investors grows. Intuitively, due to the higher effective risk-aversion of benchmarked investors and, thus, the reduction in their demand, non-benchmarked investors must be induced to tilt their portfolio toward non-index stocks, which is achieved through a higher Sharpe ratio. The positive hedging demand for the index stocks implies that less such "equilibrium incentives" are needed, so that their Sharpe ratios are lower than those of comparable non-index stocks. 
Finally, the "information gap" between the two groups of market participants widens as the fraction of benchmarked investors increases. That is, because less information is revealed through the public stock prices, the fact that non-benchmarked institutional investors invest more into information acquisition, that is, have superior private information, gains importance. This leads to a substantial out-performance of better informed, nonbenchmarked institutional investors, increasingly so, as the assets under management of benchmarked institutions grow.

Our results have important implications for the functioning of financial markets, particularly for their ability to aggregate and disseminate information and, accordingly, firms' abilities to make informed corporate decisions in the presence of benchmarked institutions. ${ }^{2}$ The findings should also be of importance for regulators in the debate about whether the "indexing industry" should be more regulated.

Our paper combines insights from two streams of the literature. First, there is a growing body of literature on the stock market implications of institutional investors. ${ }^{3}$ Brennan (1993) shows that in a static, CARA-utility setup with performance concerns relative to a benchmark, a two-factor model arises, with one of the factors being the benchmark. Cuoco and Kaniel (2011), from which we borrow the compensation scheme of institutional investors, numerically solve a model of portfolio delegation with asset managers that have CRRA-preferences and receive a linear performance fee. ${ }^{4}$ They demonstrate that symmetric fees have an unambiguously positive impact on the price of index stocks and a negative impact on their Sharpe ratios. Using a tractable specification, Basak and Pavlova (2013) provide analytical solutions for a dynamic, CRRA-utility setup with multiple risky assets and institutional investors who care about a benchmark. In their setting, institutional investors tilt their portfolio toward index stocks, hence, creating upward price pressure for

\footnotetext{
${ }^{2}$ The economic importance of price informativeness is highlighted in the literature on "feedback effects," which shows that information about fundamentals contained in asset prices affects corporate decisions. See, for example, the survey by Bond, Edmans, and Goldstein (2012) or Chen, Goldstein, and Jiang (2007), Bakke and Whited (2010), Edmans, Goldstein, and Jiang (2012), and Foucault and Frésard (2012).

${ }^{3}$ Basak and Pavlova (2016) discuss the impact of institutional investors on the commodity market.

${ }^{4}$ Cuoco and Kaniel (2011) also study asymmetric performance fees - a contract that might arise exogenously (e.g., for hedge funds) or implicitly at the fund manager's level (see the empirical evidence in Ibert, Kaniel, van Nieuwerburgh, and Vestman (2017) for Swedish mutual fund managers).
} 
these assets and an amplification of index stocks' return volatilities. Buffa, Vayanos, and Woolley (2014) study the joint determination of fund managers' contracts and equilibrium prices in a dynamic setup with multiple risky assets and CARA-preferences. Agency frictions lead to contracts that depend on managers' performance relative to a benchmark and bias the aggregate market upwards. Buffa and Hodor (2017) study the impact of heterogeneous benchmarks and document additional price pressure, which also amplifies return volatility.

In contrast to our paper, this literature focuses on the case of symmetrically informed investors. Hence, the main difference of our article from this literature is that we explicitly account for the joint information and portfolio choice of institutional investors. Interestingly, while these papers find an unambiguously positive impact of institutional investors on the prices of index assets and the aggregate market, taking into account endogenous information acquisition can lead to opposite predictions.

Second, our paper is closely related to the literature on private information and optimal information choice, starting from the early papers by Grossman (1976), Grossman and Stiglitz (1980), Hellwig (1980), and Verrecchia (1982). Part of this literature focuses on information acquisition in the asset management industry. For example, Admati and Pfleiderer (1997) study benchmarking in the compensation of privately informed portfolio managers and demonstrate that benchmark-adjusted compensation schemes are generally inconsistent with optimal risk-sharing and optimal portfolios for fund investors. García and Vanden (2009) show that competition between fund managers makes price more informative. In contrast, Qiu (2012) finds ambiguous results for price informativeness if managers' performance is evaluated against their peers. Malamud and Petrov (2014) demonstrate that convex compensation contracts lead to equilibrium mispricing but reduce price volatility. Sotes-Paladino and Zapatero (2016) find that, in partial equilibrium, a linear benchmarkadjusted compensation component can benefit investors. Kacperczyk, van Nieuwerburgh, and Veldkamp (2016) show, theoretically and empirically, that fund managers optimally choose to process information about aggregate shocks in recessions and idiosyncratic shocks 
in booms. Farboodi and Veldkamp (2016) demonstrate that, when information is scarce, fundamental information acquisition is most important, whereas order-flow analysis dominates when investors are well informed. Bond and García (2016) study the consequences of investing in risky assets only via the market portfolio and find negative externalities for uninformed investors.

In contrast to these studies, we explicitly model institutional investors' performance concerns relative to a benchmark, which allows us to make novel predictions about the relationship between the size of benchmarked institutions and information efficiency, as well as asset prices in equilibrium.

Due to our use of CRRA-preferences, which makes the equilibrium price function nonlinear, we rely on a novel, exact numerical algorithm to solve for the equilibrium. Consequently, our paper is also related to Bernardo and Judd (2000), who, however, consider only two investors rather than the continuum of investors in our framework and do not allow for heterogeneous signal precision. Our work is also related to recent studies that have relaxed the joint CARA-normal assumption ${ }^{5}$ as well as the literature on relative wealth concerns and private information (see, among others, García and Strobl (2011)).

The remainder of the paper is organized as follows. Section 1 introduces an economic setting with benchmarked institutional investors and joint portfolio and information choice. Section 2 studies a benchmarked investor's optimal portfolio and information choice problem within a single-stock, partial equilibrium version of our baseline framework. Sections 3 and 4 discuss the equilibrium implications for the single-stock and multi-stock economy, respectively. Finally, Section 5 summarizes our key predictions. Proofs, as well as a description of the numerical algorithm, are delegated to the Appendix.

\footnotetext{
${ }^{5}$ Barlevy and Veronesi (2000) and Albagli, Hellwig, and Tsyvinski (2014) study risk-neutral investors, Peress (2003) studies general preferences using a (small-risk) log-linearization, van Nieuwerburgh and Veldkamp (2010) study a general form of utility function, and Breon-Drish (2015) as well as Chabakauri, Yuan, and Zachariadis (2016) focus on distributions that are members of the "exponential family."
} 


\section{Economy with Institutional Investors and Information Choice}

In this section, we introduce our economic framework that features both-benchmarked institutional investors and a joint portfolio allocation and information choice problem. It is a static model that we break up into three (sub-)periods: the information acquisition stage

$(t=1)$, the trading stage $(t=2)$, and, finally, the consumption period $(t=3)$. We next provide the details of the economic setting.

\subsection{Economic Setting}

\section{Investment Opportunities}

There exist three financial securities that are traded competitively in the market: a riskless asset (the "bond") and two risky assets (the "stocks"). The bond pays an exogenous interest rate $r_{f}$ and is available in perfectly elastic supply. It also serves as the numéraire, with its price being normalized to one. Each stock $i$ is modeled as a claim to a random payoff $D_{i}, i \in\{1,2\}$, which is only observable in period 3 . We assume that the payoffs $D_{i}$ are independent and follow binomial distributions with high realization $D_{i, H} \equiv \mu_{D}+\sigma_{D}$ and low realization $D_{i, L} \equiv \mu_{D}-\sigma_{D}$, which are both equally likely: $\pi_{i, k}=1 / 2, k \in\{H, L\} .{ }^{6}$

The supply of each stock is assumed to be random and unobservable to prevent its price from fully revealing the information acquired by the investors and, thus, to preserve the incentives to acquire private information in the first place. Particularly, we assume that the aggregate supply of stock $i$ is given by $\bar{z}+z_{i}$ and that $z_{i}$ follows an independent, normal distribution $\mathcal{N}\left(\mu_{z}, \sigma_{z}\right)$. For example, one could think of $-z_{i}$ as the demand arising from non-institutional investors, like retail traders.

\footnotetext{
${ }^{6}$ We focus on the case of two stocks with symmetric distributions of fundamentals and noise, so that all differences between the two stocks arise from benchmarking. However, one can easily extend the setting to a case in which the fundamentals (or the noise) of the two stocks have different means or variances.
} 


\section{Information Structure}

Investors are initially, in period 1, endowed with unbiased, but uninformative, beliefs about each stock's payoff $D_{i}$. In period 1, investors can spend time and resources to acquire private information about the stocks. For example, they may study financial statements, gather information about consumers' taste, hire outside financial advisers, or subscribe to proprietary databases. Particularly, each investor $j$ can choose the precision of his private, unbiased binomial signal $S_{j, i} \in\left\{S_{L}, S_{H}\right\}$ about stock $i$ 's payoff $D_{i}$. Higher precision will reduce the conditional variance of the payoff, but will increase the information acquisition costs. Signals are assumed to be independent.

Mathematically, let $x_{j, i}$ denote the precision of investor $j$ 's signal about stock $i$ and $\rho_{j, i}$ describe the probability of a high (low) realization of the stock's payoff conditional on a high (low) realization of the signal:

$$
\rho_{j, i} \equiv \mathbb{P}_{j}\left[D_{i, H} \mid S_{j, i}=S_{H}\right]=\mathbb{P}_{j}\left[D_{i, L} \mid S_{j, i}=S_{L}\right]
$$

The precision $x_{j, i}$ then translates into probability $\rho_{j, i}$ as follows:

$$
\rho_{j, i}\left(x_{j, i}\right)=\frac{1}{2}+\frac{1}{2} \sqrt{\frac{x_{j, i}}{x_{j, i}+4}} .
$$

A signal with precision $x_{j, i} \operatorname{costs} C\left(x_{j, i}\right)$ dollars, where $C$ is increasing and strictly convex in the precision level. These assumptions guarantee the existence of an interior solution and capture the idea that each new piece of information is more costly than the previous one. Particularly, we assume that the cost function $C$ is given by

$$
C\left(x_{j, i}\right)=\kappa x_{j, i}^{c},
$$

where $\kappa$ defines the overall level of the information acquisition costs and $c>1$ describes the degree of convexity. 
Note that in case an investor chooses a precision of zero $\left(x_{j, i}=0\right)$, that is, he decides not to acquire any information about stock $i$, he receives an uninformative signal, $\rho_{j, i}(0)=1 / 2$, at zero cost $C(0)=0$. At the other extreme, perfect foresight, $\rho_{j, i}=1$, could only be achieved with infinite precision $\left(x_{j, i} \rightarrow \infty\right)$, and, thus, infinite wealth $(C(\infty) \rightarrow \infty)$.

In period 2 , investors receive their private signals (with the chosen precision), which they combine, using Bayes' law, with information from equilibrium prices to update their prior beliefs and form optimal portfolio decisions.

We denote investor $j$ 's expectation conditional on prior beliefs alone as $E_{j}[\cdot]$ and use $E_{j}\left[\cdot \mid \mathcal{F}_{j}\right]$ to denote the investor's expectation conditional on his information set $\mathcal{F}_{j}$ in period 2. Particularly, the information set contains an investor's private signals with precision $x_{j, i}$ and the publicly observable stock prices, $\mathcal{F}_{j}=\left\{S_{j, i}, P_{i} ; i \in\{1,2\}\right\}$. Note that because all probability distributions and other parameters of the economy are common knowledge, investors are only asymmetrically informed about the stocks' payoffs, $D_{i}$.

\section{Investors}

There exists a continuum of atomless investors with mass one that we separate into two heterogeneous groups of market participants, $n \in\{\mathcal{B}, \mathcal{N}\}:(1)$ a fraction $\Lambda$ of institutional investors, $\mathcal{B}$, is benchmarked ("indexed") and (2) a fraction $1-\Lambda$ of institutional investors, $\mathcal{N}$, is not benchmarked. Each investor is endowed with the same initial wealth $W_{1, j}$, which we normalize to 1 . Accordingly, $\Lambda$ represents the fraction of wealth managed by benchmarked institutional investors - or, equivalently, how large benchmarked investors are, relative to the overall economy. Varying $\Lambda$ will be our most important comparative statics analysis, because it allows us to illustrate how the growth in assets under management by indexed institutional investors influences informational efficiency and asset prices.

Motivated by the structure of asset management fees in practice and recent theoretical contributions, ${ }^{7}$ we explicitly model the compensation, $C_{j}$, of institutional investors (fund

\footnotetext{
${ }^{7}$ Basak and Pavlova (2013) demonstrate benchmarking formally using an agency-based argument. Moreover, in Buffa, Vayanos, and Woolley (2014), investors endogenously make fund managers' fees sensitive to the performance relative to a benchmark - due to agency frictions. Similarly, Sotes-Paladino and Zapatero (2016) show that a linear benchmark-adjusted component in managers' contracts can benefit investors.
} 
managers) as

$$
C_{j}\left(W_{j, 3}, R_{I}\right)=\beta_{j} W_{j, 3}+\gamma_{j} W_{1, j}\left(\left(1+R_{j, F}\right)-\left(1+R_{I}\right)\right)
$$

which is a function of, $W_{j, 3}$, the terminal wealth and, $R_{I}$, the return on the "index" against which the performance of the institutional investor is measured (the benchmark portfolio). We define the investor's portfolio return as $R_{j, F} \equiv \frac{W_{j, 3}}{W_{1, j}}-1=W_{j, 3}-1$. We assume that $\beta_{j} \geq 0$ as well as $\gamma_{j} \geq 0$ and $\beta_{j}+\gamma_{j}>0$, so that buying the benchmark is always a feasible strategy that yields a strictly positive compensation. ${ }^{8}$

Thus, investors' compensation can have two components; first, a management fee, $\beta W_{j, 3}$, that is proportional to the terminal value of the fund portfolio; second, a performance fee, $\gamma_{j}\left(\left(1+R_{j, F}\right)-\left(1+R_{I}\right)\right)$, that is proportional to the portfolio return in excess of the return of the index, with $\gamma_{j}$ denoting the degree of benchmarking. ${ }^{9}$ These types of fees are known as fulcrum performance fees. The 1970 Amendment of the Investment Advisers Act of 1940 restricts mutual fund fees to be of the fulcrum type.

For ease of exposition, we set $\beta_{\mathcal{B}}=\beta_{\mathcal{N}}=\beta$, so that the only source of heterogeneity across the two groups of investors is the degree of benchmarking, $\gamma_{n}$. Particularly, while for benchmarked investors the degree of benchmarking is assumed to be above zero, $\gamma_{\mathcal{B}}>0$, we shut down benchmarking for the group of non-benchmarked investors, $\gamma_{\mathcal{N}}=0$.

The objective of each investor $j$ is to maximize his expected utility over time-3 compensation, $C_{j}$, with the preferences being represented by power utility with risk-aversion $\alpha:$

$$
U_{j}\left(W_{j, 3}, R_{I}\right)=\frac{C_{j}\left(W_{j, 3}, R_{I}\right)^{1-\alpha}}{1-\alpha}
$$

In Appendix A we prove the following Lemma:

\footnotetext{
${ }^{8}$ This is true as long as the index return exceeds $-100 \%$, i.e., the final payoff exceeds zero.

${ }^{9}$ An institutional investor's desire to perform well relative to a benchmark may also be driven by social status, associated with a fund's performance relative to the index, instead of monetary incentives.
} 
Lemma 1. The local curvature of the institutional investors' utility function, that is, the effective (local) risk-aversion, $\hat{\alpha}_{j}$, is given by

$$
\hat{\alpha}_{j} \equiv-\frac{W_{j, 3} \partial^{2} U_{j} / \partial W_{j, 3}^{2}}{\partial U_{j} / \partial W_{j, 3}}=\alpha\left(1-\frac{\gamma_{j}}{\beta_{j}+\gamma_{j}}\left(\frac{1+R_{j, F}}{1+R_{I}}\right)^{-1}\right)^{-1}
$$

If the return on the benchmark, $R_{I}$, exceeds -1 , and $\gamma_{j}>0$, it holds that

$$
\hat{\alpha}_{j}>\alpha ; \quad \frac{\partial \hat{\alpha}_{j}}{\partial \gamma_{j}}>0 ; \quad \frac{\partial \hat{\alpha}_{j}}{\partial\left(\left(1+R_{j, F}\right) /\left(1+R_{I}\right)\right)}<0 .
$$

Lemma 1 shows that the effective risk-aversion of a benchmarked investor $\left(\gamma_{j}>0\right)$ exceeds the risk-aversion of a non-benchmarked investor (which is equal to $\alpha$ ) and is increasing in the degree of benchmarking $\gamma_{j}$. Moreover, a low "surplus performance" $\left(1+R_{j, F}\right) /\left(1+R_{I}\right)$ implies a high local risk-aversion. ${ }^{10}$ This behavior of the local risk-aversion in the presence of relative performance concerns shares many similarities with the local risk-aversion for external habit (see Campbell and Cochrane (1999)), which is increasing as an investor's surplus consumption declines.

REMARK 1. Our specification of the benchmarked investors' compensation scheme exhibits three important characteristics that let them behave differently from non-benchmarked investors. First, benchmarked investors care about their performance relative to a benchmark. Second, benchmarked investors have an incentive to post a high return when their benchmark is high or, formally, their marginal utility of wealth is increasing in the index return $R_{I}$. Third, the benchmarked investors' utility function is decreasing in the index return $R_{I}$.

REMARK 2. Our definition of the investors' compensation scheme (2) closely resembles the fee structure in Cuoco and Kaniel (2011), with the exception that we do not incorporate a constant "load fee," which is independent of assets under management and performance. This load fee is, however, set to zero for most of the analysis in Cuoco and Kaniel (2011)

\footnotetext{
${ }^{10}$ One can derive a similar formula for the case of CARA-utility, which is, however, a bit more simplistic because, due to the absence of wealth effects, the local risk-aversion will only depend on the degree of benchmarking and not on the surplus performance.
} 


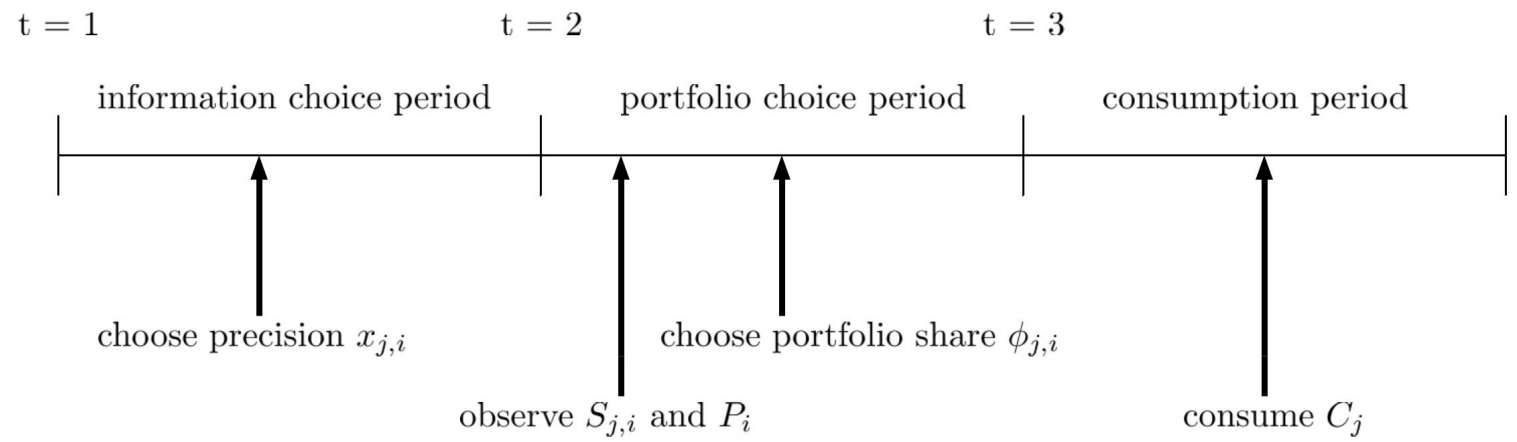

Figure 1: Timing. The figure illustrates the timing of the events.

anyway. Moreover, the investors' utility function shares many similarities with the specification in Basak and Pavlova (2013), ${ }^{11}$ with the notable exception that, in contrast to their tractable specification, our utility is decreasing in the benchmark. This is crucial, because in contrast to asset pricing models with symmetric information in which only marginal utility matters, the investors' utility functions play a key role in models of information acquisition.

\section{Timing}

There exist three (sub-)periods. In period 1, the information acquisition stage, each investor chooses the precision $x_{j, i}$ of his private signals about the stocks' payoffs, subject to an increasing cost $C\left(x_{j, i}\right)$ for more precise information. In period 2 , the trading stage, each investor observes his private signals. At the same time, financial markets open and investors observe the equilibrium stock prices, which act as public signals. Each investor combines the public and private signals to form his posterior beliefs and, accordingly, determines how much to invest into the stocks. In period 3 , the consumption period, investors receive asset payoffs and realize utility. Figure 1 illustrates the sequence of the events.

\footnotetext{
${ }^{11}$ With $\beta_{j}+\gamma_{j}=1$, it is suggested as an alternative specification (see Remark 1 in Basak and Pavlova (2013)).
} 


\section{Posterior Beliefs}

Investors in the economy can submit demand schedules, that is, condition their demand on the prices of the stocks. Hence, they can learn from equilibrium prices, which imperfectly reveal information about the other investors' private signals. Particularly, any investor in the economy who receives an informative signal about one of the stocks will use his private information to optimize his portfolio and buy more or less of the specific stock, depending on the signal realization. Because the supply of the stock is limited, the investor's demand will move the price, and, hence, his private information will get incorporated into the stock price. Consequently, any rational investor will use the stock prices together with his private signals to form his posterior beliefs about the stocks' payoffs.

Note that because the stocks' payoffs, the signals, and the noise are independent, we can compute the investors' posterior beliefs for the two stocks independently as well. Because each investor is small, the distribution the private signals and, accordingly, aggregate demand depends exclusively on the underlying payoff $D_{i}$ (see Hellwig (1980)), so that the price is a function of a stock's payoff and its supply-both unobservable - only: $P_{i}\left(D_{i}, z_{i}\right){ }^{12}$ Consequently, for a given price $P_{i}$, each investor can back out the two combinations of payoff and noise, denoted by $\left\{\left(D_{i, L}, z_{i, L}\right),\left(D_{i, H}, z_{i, H}\right)\right\}$, which are consistent with this price. ${ }^{13}$ For example, a high stock price could be due to a high underlying payoff or a low supply. Using the distribution of the noise, an investor can then compute the posterior probability of the payoff $D_{i}$.

Formally, investor $j$ 's posterior probability of a payoff realization $D_{i, k}, k \in\{L, H\}$, is given by

$$
\hat{\pi}_{k, j, i}=\mathbb{P}\left(D_{i, k} \mid P_{i}, S_{j . i}\right)=\frac{f_{z}\left(z_{i, k}\right) \mathbb{P}\left(D_{i, k} \mid S_{j, i}\right)}{\sum_{d} f_{z}\left(z_{d}\right) \mathbb{P}\left(D_{i, d} \mid S_{j, i}\right)}
$$

\footnotetext{
${ }^{12}$ Note that if the supply were not random, the stock price would be a function of the payoff only. Accordingly, there would only be a single payoff realization consistent with a given price, so that prices would be fully revealing. In this case, there would be no trading in equilibrium (Milgrom and Stokey (1982)) and no incentives to acquire private information in the first place, so that no competitive equilibrium would exist (Grossman and Stiglitz (1980)).

${ }^{13}$ The supply $z_{i, k}, k \in\{L, H\}$, is simply given by the aggregate demand in the economy at price $P_{i}-$ conditional on $D_{k, i}$. See also the descriptions and derivations in Breugem (2016) for learning from price in a dynamic setting.
} 
where $f_{z}(\cdot)$ denotes the density function of the normally distribution noise $z_{i}$, and $\mathbb{P}\left(D_{i, n} \mid S_{j . i}\right)$ can be computed directly as

$$
\mathbb{P}\left(D_{i, d} \mid S_{j, i}\right)=\frac{\mathbb{P}\left(D_{i, d}, S_{j, i}\right)}{\sum_{m} \mathbb{P}\left(D_{i, m}, S_{j, i}\right)}
$$

using the correlation $\rho_{j, i}$ between investor $j$ 's private signal and the payoff $D_{i}$ :

$$
\mathbb{P}\left(D_{i, m}, S_{j, i}\right)=\left\{\begin{array}{ll}
\rho_{j, i} / 2 & \text { if } m=j, \\
\left(1-\rho_{j, i}\right) / 2 & \text { if } m \neq j ;
\end{array} \quad \text { with } m, j \in\{L, H\}\right.
$$

\section{$1.2 \quad$ Numerical Illustration}

Our numerical illustrations are based on the following set of parameters: The mean and volatility of the stocks' payoffs, $\mu_{D}$ and $\sigma_{D}$, are set to 1.05 and 0.25 , respectively. The riskfree rate, $r_{f}$, is set to zero. Investors have relative risk-aversion, $\alpha$, of 3 and the proportional component of the compensation scheme, $\beta$ is set to $2 \%$. Investors are endowed with an initial wealth, $W_{1, j}$, of 1 . We assume a quadratic information acquisition cost function $(c=2)$ with $\kappa=0.01$. Finally, we assume that the mean and volatility of the normal distribution governing the noisy supply, $z_{i}, i \in\{1,2\}$, are given by $\bar{z}=0.8$ and $\sigma_{z}=0.20$, which guarantees some realistic Sharpe ratios. Table 1 provides a summary of the parameters.

\section{Portfolio and Information Choice of Benchmarked Investors}

We start our analysis within the framework of a single-stock economy. The main reason for this is expositional simplicity. Particularly, it turns out that many of the key insights of the paper can already be explained within this framework. Accordingly, we will refer to the single stock, which also serves as the benchmark, as the "stock market" and for ease of notation drop the subscript $i$. In Section 4 we expand the framework to demonstrate how our results generalize in a multi-stock economy. 


\begin{tabular}{clc}
\hline \hline Variable & Description & Value \\
\hline & & \\
$\mu_{D}$ & Mean of stock payoff $D$ & 1.05 \\
$\sigma_{D}$ & Volatility of stock payoff $D$ & 0.25 \\
$r_{f}$ & Risk-free rate & 0 \\
$\alpha$ & Relative risk-aversion & 3 \\
$\beta$ & Compensation scheme: proportional component & 0.02 \\
$W_{1, j}$ & Initial wealth & 1 \\
$c$ & Exponent of information cost function & 2 \\
$\kappa$ & Level of information cost function & 0.01 \\
$\bar{z}$ & Mean of noisy supply & 0.80 \\
$\sigma_{z}$ & Volatility of noisy supply $z_{i}$ & 0.20 \\
\hline
\end{tabular}

Table 1: Model Parameters. This table reports the parameter values used for our numerical illustrations.

Moreover, to provide the intuition for the main economic mechanisms that drive our equilibrium results in the clearest possible way, we first analyze an economy of the "partial equilibrium" type, that is, with an exogenous price. Within this setting, we build our basic intuition for a benchmarked investor's portfolio allocation and information choice.

Specifically, an investor's optimization problem follows the timing illustrated in Figure 1 and must be solved in two stages - working backward from the trading period $(t=2)$, in which an investor chooses his optimal portfolio, to the information acquisition stage $(t=1)$, in which he determines the optimal signal precision.

\subsection{Portfolio Choice}

Given an investor's posterior beliefs, described by his information set $\mathcal{F}_{j}$, he chooses the fraction of wealth to be in the form of stocks, $\phi_{j}$, to maximize his expected utility, taking the price $P$ as given:

$$
V\left(S_{j}, x_{j}, W_{j, 2} ; P\right)=\max _{\phi_{j}} E_{j}\left[U_{j}\left(W_{j, 3}, R_{I}\right) \mid \mathcal{F}_{j}\right]
$$


where $V\left(S_{j}, x_{j}, W_{j, 2} ; P\right)$ denotes the value function. Denoting the stock market's excess return by $R^{e} \equiv \frac{D-P}{P}-r_{f}$, the optimization is subject to the following budget equation:

$$
W_{j, 3}=W_{j, 2}\left(1+r_{f}+\phi_{j} R^{e}\right) .
$$

Substituting wealth $W_{j, 3}$ into the optimization problem (6), the first-order condition with respect to $\phi_{j}$ yields the optimal portfolio:

$$
E_{j}\left[U_{j}^{\prime}\left(W_{j, 2}\left(1+r_{f}+\phi_{j} R^{e}\right), R_{I}\right) W_{j, 2} R^{e} \mid \mathcal{F}_{j}\right]=0
$$

where $U_{j}^{\prime}(\cdot)$ denotes the derivative of the investor's utility function with respect to wealth. Denoting the first two components as the stochastic discount factor, equation (8) gives the usual asset pricing interpretation that the "price" of the excess return $R^{e}$ has to be zero.

Because of the complexities introduced by CRRA-utility, we solve the model numerically. However, to provide some clear intuition for one of our key findings, we derive in Appendix A the following closed-form, approximate solution for the fraction of wealth invested into the stock market:

$$
\phi_{j} \approx \frac{1}{\alpha} \frac{E_{j}\left[R^{e} \mid \mathcal{F}_{j}\right]}{\operatorname{Var}_{j}\left[R^{e} \mid \mathcal{F}_{j}\right]}+\frac{\gamma_{j}}{\beta_{j}+\gamma_{j}}-\frac{\gamma_{j} \xi_{j}}{\alpha\left(1+\gamma_{j} \xi_{j}\right)} \frac{E_{j}\left[R^{e} \mid \mathcal{F}_{j}\right]}{\operatorname{Var}_{j}\left[R^{e} \mid \mathcal{F}_{j}\right]},
$$

where $\xi_{j}=E\left[\frac{1+R_{j, F}}{1+R_{I}} \mid \mathcal{F}_{j}\right]$ denotes the expected surplus performance.

That is, a benchmarked investor's $\left(\gamma_{j}>0\right)$ portfolio has three components. The first component is the standard mean-variance efficient portfolio, which is independent of the degree of benchmarking. It is the same portfolio that a non-benchmarked investor holds. The second component is a hedging portfolio, which arises because a benchmarked investor has an incentive to do well when the index does well, which can be achieved by buying assets that co-vary positively with the benchmark. In the single-asset economy, the stock market, which also serves as the benchmark, naturally co-varies positively with the benchmark. 
This results in an additional demand, $\frac{\gamma_{j}}{\beta_{j}+\gamma_{j}}>0$, which is increasing in the degree of benchmarking. This component is information-insensitive, that is, not affected the investor's posterior beliefs. Intuitively, it is, by definition, designed to closely track or, formally, covary with, the benchmark, and not designed for speculation.

The third component captures the change in the investor's risk attitude resulting from relative performance concerns. Particularly, as shown in Lemma 1, the investor's effective risk-aversion is increasing in the degree of benchmarking. As long as the expected excess return on the market is positive, an increase in risk-aversion implies a reduction in the fraction invested into the stock market, that is, the third component is negative and decreasing (becoming more negative) in the degree of benchmarking. As we will shortly see, this component is the key driver of many results in the paper.

One can rewrite the fraction of wealth invested into the stock market in (9) as

$$
\phi_{j} \approx\left(\frac{1}{\alpha}-\frac{\gamma_{j} \xi_{j}}{\alpha\left(1+\gamma_{j} \xi\right)}\right) \frac{E_{j}\left[R^{e} \mid \mathcal{F}_{j}\right]}{\operatorname{Var}_{j}\left[R^{e} \mid \mathcal{F}_{j}\right]}+\frac{\gamma_{j}}{\beta_{j}+\gamma_{j}}
$$

which shows explicitly that the portfolio is composed of the standard mean-variance efficient portfolio of an investor with risk-aversion $\hat{\alpha}$, as in (4), and a hedging portfolio.

We can now study the impact of benchmarking on the institutional investor's portfolio for the illustrative setting described in Section 1.2. ${ }^{14}$ We start with the case of an uninformed investor $\left(x_{j}=0\right)$, which illustrates the impact of benchmarking in the absence of private information. Panel A of Figure 2 shows that the fraction of wealth invested into the stock market is increasing in the degree of benchmarking, leading to an excess demand for the stock market. This implies that the positive hedging demand dominates the drop in demand, resulting from the increase in effective risk-aversion.

This result is fully consistent with the asset pricing literature that studies institutional investors with symmetric information. For example, Brennan (1993), Cuoco and Kaniel (2011), Basak and Pavlova (2013), and Buffa, Vayanos, and Woolley (2014) find that insti-

\footnotetext{
${ }^{14}$ To closely connect the results to the equilibrium results that follow, we use the equilibrium price for the portfolio allocation problem.
} 
tutions optimally tilt their portfolios toward stocks that are included in their benchmark index. Moreover, because this is the typical pattern in equilibrium, we will exclusively focus on this case going forward. ${ }^{15}$

Panel A of Figure 2 also depicts the expected portfolio share of the stock market for an informed investor $\left(x_{j}>0\right)$. As expected, the informed investor invests, on average, more into the stock market -irrespective of the degree of benchmarking. Intuitively, keeping $\gamma_{j}$ fixed, the conditional variance $\operatorname{Var}_{j}\left[R^{e} \mid \mathcal{F}_{j}\right]$ is lower for a better informed investor. This increases the risk-adjusted return, and, in turn, increases the standard mean-variance component of the portfolio relative to an uninformed investor. Because the hedging portfolio is information-insensitive, this leads to an increase in the expected portfolio share of the market. The additional demand needs to be financed. Accordingly, as Panel B of Figure 2 illustrates, the portfolio share of the bond, $1-\phi_{j}$, is declining in the degree of benchmarking - for the uninformed and the informed investor, with the difference being more pronounced for the informed investor because of the stronger demand for the stock market.

In contrast to the uninformed investor, the informed investor's posterior beliefs and, in turn, his investment decisions, depend on the signal realization $S_{j} \in\left\{S_{L}, S_{H}\right\}$. Panel C of Figure 2 shows that, as expected, an informed, non-benchmarked investor $\left(\gamma_{j}=0\right)$ overweights the stock market in his portfolio following a positive signal, $S_{H}$, and under-weights the stock market following a negative signal, $S_{L}$. Intuitively, Bayesian learning leads to a high conditional expected return $E_{j}\left[R^{e} \mid \mathcal{F}_{j}\right]$ following a positive signal, which increases the standard mean-variance demand in equation (10), and vice versa for a negative signal. Consequently, a non-benchmarked institutional investor under-weights the bond following a positive signal and over-weights it following a negative signal (see Panel D).

We now focus on the interaction between benchmarking and speculation based on private information. As Panel C of Figure 2 illustrates, for a signal with given precision, the spread between a benchmarked investor's conditional stock demand following a positive and a

\footnotetext{
${ }^{15}$ It is, however, theoretically possible that the risk reduction effect dominates, and the demand of the benchmarked investors is lower than the demand of the non-benchmarked investors.
} 
A: Expected Stock Demand

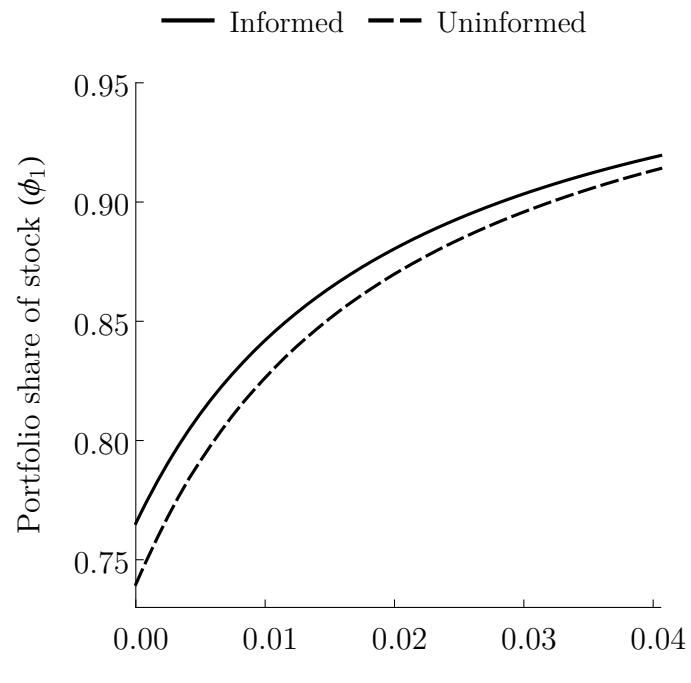

Degree of benchmarking $(\gamma)$

C: Conditional Stock Demand

-ー $S_{H}=-S_{L} \longrightarrow$ Expected

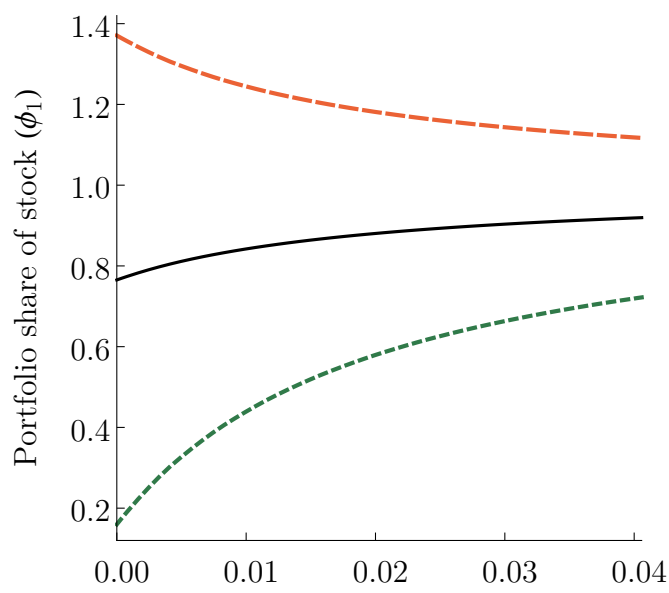

Degree of benchmarking $(\gamma)$
B: Expected Bond Demand

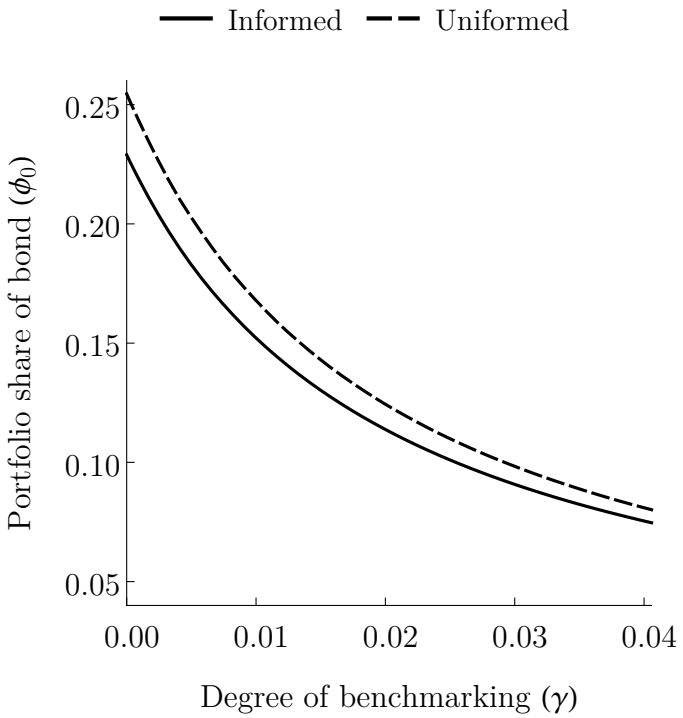

D: Conditional Bond Demand

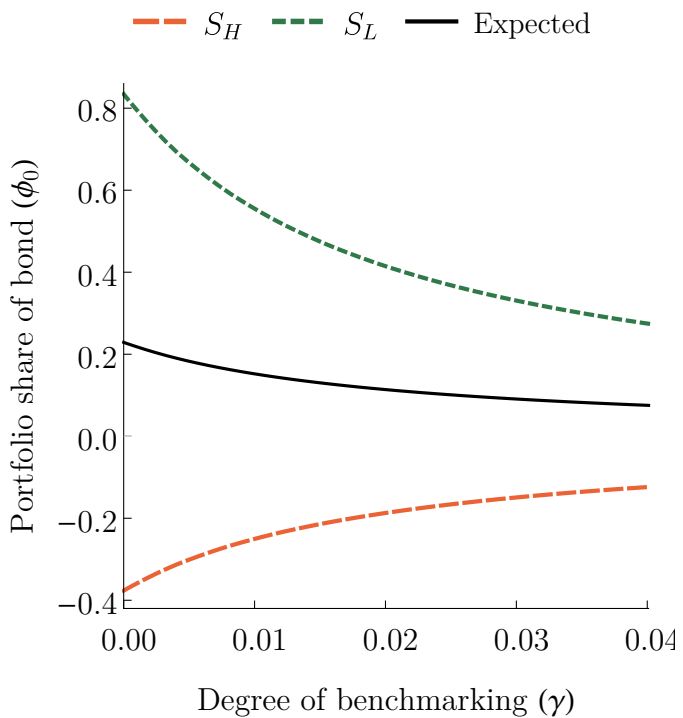

Figure 2: Asset Demand. The figure depicts the optimal period-2 portfolio choice of a single institutional investor, who takes the stock price $P$ and the precision of his signal $x_{j}$ as given, as a function of the degree of benchmarking $\gamma$. Panels A and B show the expected portfolio share of the stock and the bond of an "uninformed" investor with a signal precision of zero $\left(x_{j}=0\right)$ and an informed investor who receives an private signal with $x_{j}>0$. Panels $\mathrm{C}$ and D show the portfolio share of the stock and the bond for the same informed investor-conditional on the realization of his signal $S_{j} \in\left\{S_{H}, S_{L}\right\}$. The results are based on the parameter values presented in Table 1.

negative signal narrows. That is, in the presence of benchmarking, the investor speculates less, with the effect strengthening with the degree of benchmarking $\gamma_{j}$. For example, an 
institutional investor who is concerned about his performance relative to a benchmark has a lower demand for the stock following a positive signal than an investor who is not benchmarked. In Panel $\mathrm{D}$ one can observe offsetting effects in the bond holdings of the benchmarked investor.

Intuitively, this decline in the speculative activities of a benchmarked investor in reaction to private information is driven by the increase in the benchmarked investor's effective risk-aversion. A higher effective risk-aversion implies that the investor will take smaller bets. The effect is also apparent from the expression for the portfolio share of the stock in equation (9). Particularly, one can easily show that the third (negative) component, capturing the change in risk aversion, is decreasing (becoming more negative) in the degree of benchmarking, which reduces the sensitivity of the stock market's portfolio share with respect to the conditional expected excess return (see also (10)). The asymmetry in the "slopes" of the benchmarked investor's conditional stock market demand is driven by the underlying positive hedging demand, which is information-insensitive and, thus, always creates a positive demand.

\subsection{Information Choice}

Having determined the impact of benchmarking on an institutional investor's portfolio choice in period 2, that is, after observing the private signal, we can now study his optimization problem in period 1 - the information acquisition stage. At this point, the investor needs to choose the precision, $x_{j}$, of the private signal that he will receive in period 2 , anticipating his optimal portfolio choice in period 2 in reaction to a signal realization with chosen precision.

Formally, the investor chooses the precision $x_{j}$ in order to maximize his unconditionally expected utility, based on prior information only, of the period-2 value function, taking $W_{1, j}$ as given:

$$
\max _{x_{j} \geq 0} E_{j}\left[V\left(S_{j}, x_{j}, W_{j, 2} ; P\right)\right]
$$


A: Signal Precision

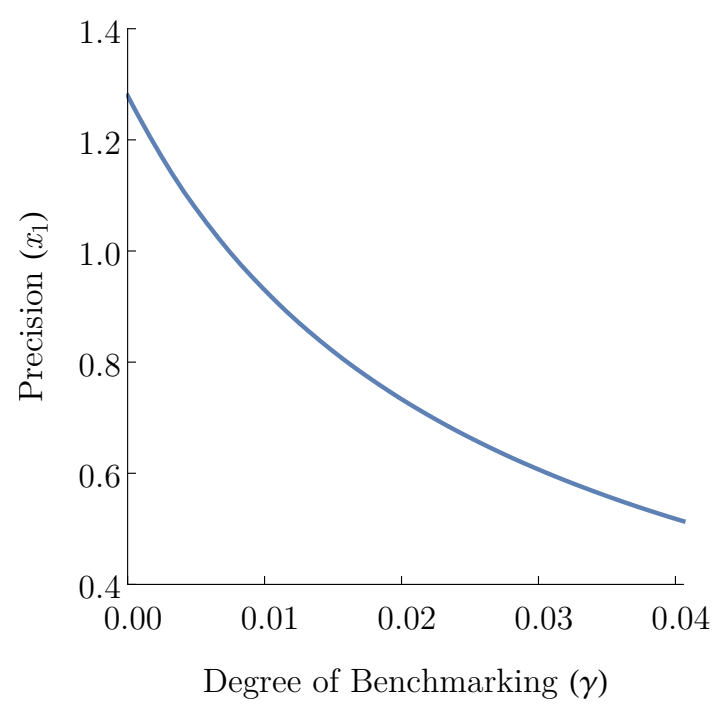

B: Variance explained by Signal

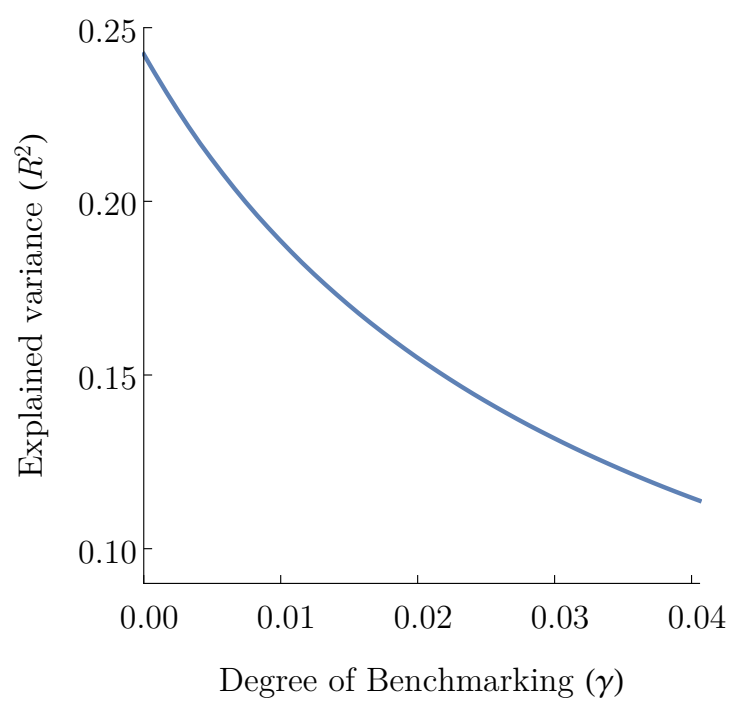

Figure 3: Information Demand. The figure shows the information choice of a single institutional investor in period 1 as a function of the degree of benchmarking $\gamma$. Panels A and B depict the signal precision, $x_{j}$, chosen by the institutional investor and the fraction of the variance of the payoff that is explained by a signal with chosen precision, $R^{2}$, respectively. The results are based on the parameter values presented in Table 1.

subject to the budget equation

$$
W_{j, 2}=W_{1, j}-C\left(x_{j}\right) .
$$

Substitution wealth $W_{j, 2}$ into the optimization problem (11), the first-order condition with respect to $x_{j}$ yields the optimal signal precision

$$
E_{j}\left[\frac{\partial V\left(S_{j}, x_{j}, W_{j, 2} ; P\right)}{\partial x_{j}}-\frac{\partial V\left(S_{j}, x_{j}, W_{j, 2} ; P\right)}{\partial W_{j, 2}} \frac{\partial C\left(x_{j}\right)}{\partial x_{j}}\right]=0
$$

In expectation, an investor equates the marginal benefit of information, arising from more precise posterior beliefs and, accordingly, a better portfolio choice, to the marginal cost of information, arising from the information cost function $C\left(x_{j}\right)$.

As shown in Panel A of Figure 3, the precision that an institutional investor chooses for his private signal is declining monotonically in the degree of benchmarking. That is, he is 
less willing to invest into the acquisition of private information in period 1 and, consequently, the signal that he will receive in period 2 will be less precise.

To understand this effect, recall from the preceding section that an investor's speculative activities decline with the degree of benchmarking, due to the rise in his effective riskaversion. He is less willing to take large bets, and, thus, his portfolio choice becomes less sensitive to the realization of his signal. That means a signal with the same precision $x_{j}$ is incorporated to a smaller degree into the portfolio of a benchmarked investor than into the portfolio of an investor who is not benchmarked. Consequently, keeping signal precision unchanged, a signal is less valuable for a benchmarked investor because it has a smaller positive impact on his portfolio choice and, in turn, his compensation, as well as period-2 utility. This has a direct impact on the optimal choice of the signal precision in period 1. It renders a benchmarked investor's period-1 expected utility less sensitive to the precision of the signal, so that the investor is less willing to costly acquire information; that is, he optimally chooses a lower precision. These effects strengthen with the degree of benchmarking, which explains the decline of signal precision.

To provide some intuition on the magnitude of the signal precision $x_{j}$, Panel B of Figure 3 also shows the $R^{2}$, that is, the fraction of the variance of the payoff $D$ that is explained by the investor's signal - as a function of the degree of benchmarking. The $R^{2}$ is one-toone related to the signal precision through the investor's posterior and can be expressed as $R^{2}=1-4 E\left[\hat{\pi}_{1, j} \hat{\pi}_{2, j}\right]$, where $\hat{\pi}_{\cdot, j}$ denotes the posterior probability, as specified in (5). Accordingly, as the $R^{2}$ is monotone in $\hat{\pi}$, it is also declining in the degree of benchmarking.

\section{Information Acquisition and Asset Prices in Equilibrium}

We now focus on how benchmarking affects informational efficiency and asset prices in equilibrium - still within the framework of a one-stock economy. Instead of a single institutional investor, we consider a continuum of atomless investors and impose market clearing. Thus, each investor can extract information about the other investors' private information from the price, which will, in turn, affect his portfolio and information choice. 
Specifically, we fix the degree of benchmarking for the group of benchmarked investors $\mathcal{B}$ at $\gamma_{\mathcal{B}}=1.0 \%\left(\gamma_{\mathcal{N}}=0\right)$. Instead, our main comparative statics parameter will be the fraction of benchmarked investors, $\Lambda$. This will allow us to illustrate how the rise of "indexed" investors (or, more precisely, the growth in their assets under management) influences asset prices and informational efficiency.

\subsection{Equilibrium}

A rational expectations equilibrium is defined as a set of asset demands $\left\{\phi_{j, i}\right\}$ and information choices $\left\{x_{j, i}\right\}$ for all investors $j$ and price functions $\left\{P_{i}\right\}$ such that three conditions are satisfied

1. $\left\{x_{i, j}\left(W_{1, j}\right)\right\}$ and $\left\{\phi_{j, i}\left(S_{j, i}, x_{j, i}, W_{j, 2} ; P_{i}\right)\right\}$ solve investor $j$ 's maximization problems, given in (6) and (11), taking prices $P_{i}$ as given.

2. Each investor has rational expectations, $E_{j}\left[\cdot \mid \mathcal{F}_{j}\right]$, formed according to (5)-conditioning on the public stock prices $P_{i}$ and his private signals $S_{j, i}$ with precision $x_{j, i}$.

3. $P_{i}$ clears the market, that is, aggregate demand equals aggregate supply:

$$
\int_{0}^{1} \phi_{j}\left(S_{j}, x_{i, j}, W_{j, 2} ; P_{i}\right) \frac{W_{j, 2}}{P_{i}} d j=\bar{z}+z_{i}
$$

In equilibrium, the stock prices play a dual role: They clear the security market for each stock and aggregate as well as disseminate investors' private information. Because of our deviation from the standard CARA-normal framework, the price functions are nonlinear, so that we have to rely on a numerical algorithm to solve for the equilibrium. Appendix B provides details on the algorithm.

\subsection{Information Acquisition and Price Informativeness}

In a first step, we study how changes in the fraction of benchmarked investors affect the optimal information acquisition of the two groups of market participants, that is, the precision that investors choose for their private signal. As is shown in Panel A of Figure 4, both 
A: Informativeness of Private Signals

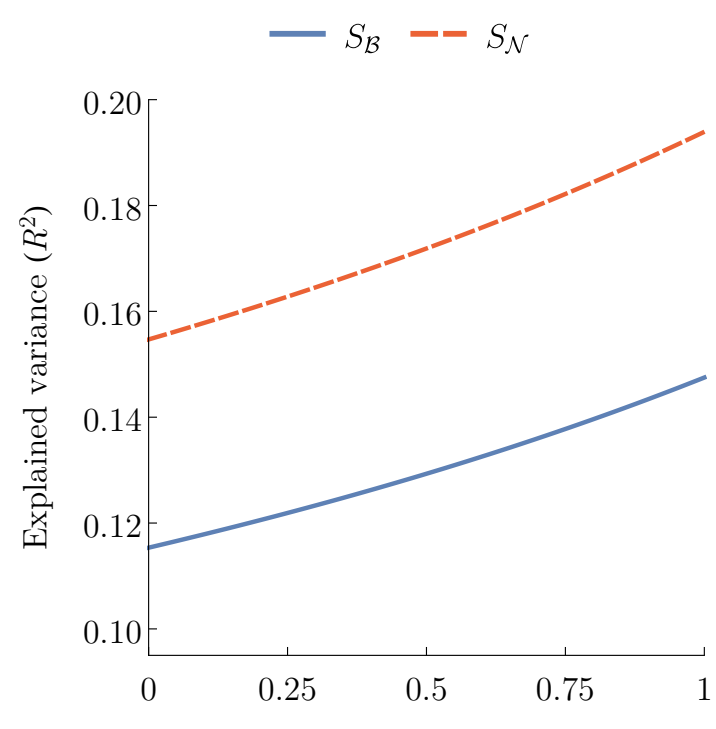

Fraction of benchmarked investors $(\Lambda)$
B: Price Informativeness

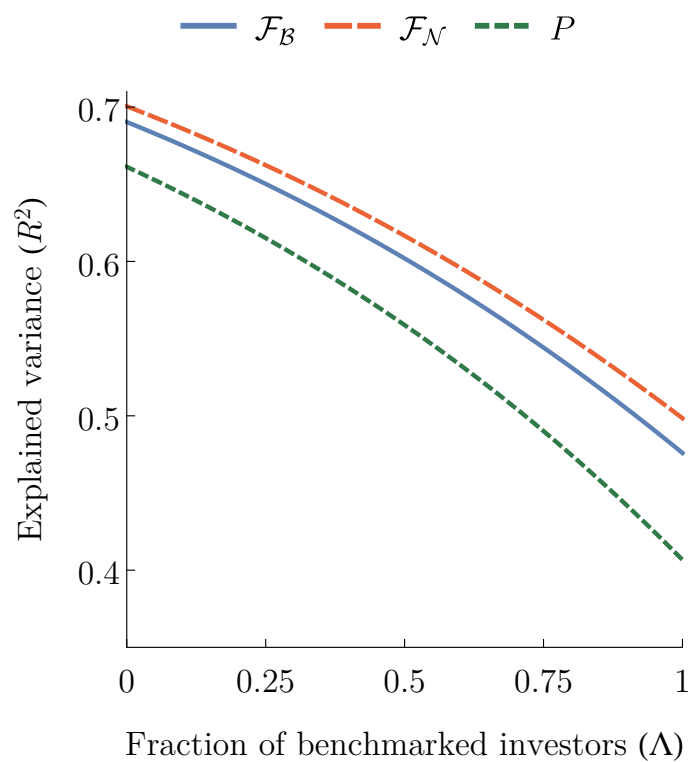

Figure 4: Informativeness of Private Signals and the Price. This figure shows the fraction of the variance of the payoff $D$ that is explained by various information sets $\left(R^{2}\right)$ as a function of the share of benchmarked investors $\Lambda$. Panel A shows the fraction of the variance that is explained by the individual investors' private signals $S_{n}$, and Panel B shows the fraction of the variance that is explained by the stock price, $P$, as well as by the two groups of market participants' information sets, $\mathcal{F}_{n}=\left\{P, S_{n}\right\}, n \in\{\mathcal{B}, \mathcal{N}\}$. The results are based on the parameter values presented in Table 1.

groups of investors choose a higher precision for their private signals, $x_{n}, n \in\{\mathcal{B}, \mathcal{N}\}$ - here again expressed in terms of $R^{2}$ —as the share of benchmarked investors increases.

To understand this effect, recall from the preceding section that benchmarking increases investors' effective risk-aversion and, thus, limits their speculative activities. Hence, benchmarked investors, in general, value private information less and, thus, choose less precise signals. For example, in Panel A of Figure 4, the variance explained by the benchmarked investors' signals $S_{\mathcal{B}}$ (or, implicitly, the signal precision) is always lower than for the nonbenchmarked investors - regardless of the share of benchmarked investors. Now, imagine an increase in the fraction of benchmarked investors without a change in the precision of the investors' private signals. This would imply a shift toward less informed benchmarked investors and, in turn, a decline in aggregate information acquisition in the economy. Accordingly, the marginal benefit from an additional piece of information goes up, increasing the incentives for all investors to acquire more information, that is, choose a more precise 
signal. This is exactly the "on-equilibrium" behavior of the two groups of investors, as shown in Panel A of Figure 4.

In equilibrium, financial markets aggregate private information through means of trading and market clearing. Accordingly, the equilibrium price partially reveals private information. Panel B of Figure 4 illustrates that price informativeness, measured as the fraction of the variance of the payoff of the stock that can be explained by the price $P$ alone, is declining in the share of benchmarked investors. This means that prices become less informative, as a bigger share of wealth is managed by benchmarked investors.

Intuitively, this effect can be explained by the fact that an increase in the share of benchmarked investors implies that better informed, non-benchmarked investors are replaced by less informed benchmarked investors. Thus, on aggregate, less information is acquired and, because benchmarked investors make smaller bets, incorporated into the price. Hence, less information is revealed through the price. The increase in the signal precision of each group of investors counteracts this effect but is not strong enough to overturn it, leading to the observed decline in price informativeness.

Panel B of Figure 4 also shows the fraction of the variance of the stock market's payoff that can be explained by the full information set of each investor, $\mathcal{F}_{n}=\left\{P, S_{n}\right\}$, consisting of the publicly observable price $P$ and his private signal $S_{n}, n \in\{\mathcal{B}, \mathcal{N}\}$. As demonstrated above, benchmarked investors always - independent of their size relative to the overall economy - choose a lower signal precision than the other investors, so it is not surprising that they are less well informed. That is, the variance of the stock market's payoff explained by benchmarked investors' information set $\mathcal{F}_{\mathcal{B}}$ is lower than the variance explained by the other investors' information set $\mathcal{F}_{\mathcal{N}}$.

Interestingly, the "information gap" between the two market participants' information sets widens as benchmarked investors constitute a larger fraction of the economy. The information advantage of the non-benchmarked investors strengthens. This is a direct consequence of the decline in price informativeness. That is, because less information is revealed through the public signal - the stock price - the precision of the investors' private 


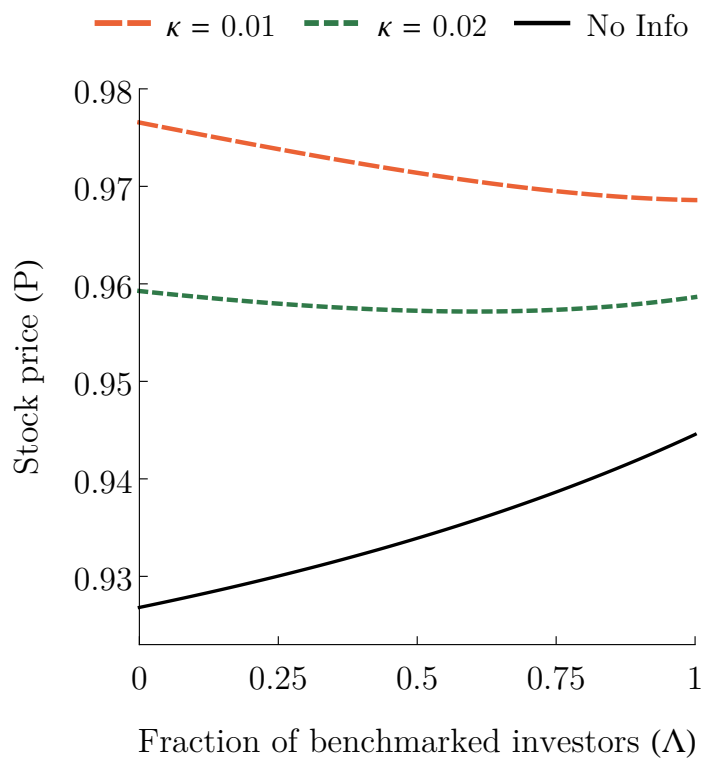

Figure 5: Stock Price. The figure shows the equilibrium expected price as a function of the share of benchmarked investors $\Lambda$. The price is depicted for the case without information acquisition ("No info"), i.e., with uninformed investors, as well as for the case with information acquisition, i.e., endogenous signal precision. It is shown for two levels of the information cost: $\kappa=0.01$ and $\kappa=0.02$. The results are based on the parameter values presented in Table 1.

information gains importance. Hence, the fact that the benchmarked investors acquire less information, that is, have less precise signals, plays a more important role.

\subsection{Asset Prices and Moments of Return}

Next, we analyze the impact on the stock price and the corresponding moments of the return in equilibrium.

Figure 5 depicts the expected equilibrium price of the market as a function of the share of benchmarked investors. In the case without information acquisition, the price is increasing in the fraction of benchmarked investors. Particularly, recall that relative performance concerns lead to a positive hedging demand for the stock market from benchmarked investors. Intuitively, this excess demand generates "price pressure." The stock is in fixed supply, so its price must increase. This finding is similar to the results in standard asset pricing models with institutional investors absent information acquisition, as in Cuoco and Kaniel (2011), Basak and Pavlova (2013) and Buffa, Vayanos, and Woolley (2014). 
Before turning to the implications of jointly modeling benchmarked investors and information acquisition, note that in rational expectations models, private information and the public information revealed through the price render investments into the stock market less risky to investors, because more precise information about its payoff is available. Therefore, risk-averse investors value the asset more. Accordingly, in the absence of benchmarked investors $(\Lambda=0)$, a higher price prevails in the economy with endogenous information choice, compared to the economy with uninformed investors.

This mechanism also explains why the price of the stock market can decline in the fraction of benchmarked investors, as shown in Figure 5 for information costs of $\kappa=0.01$. On the one hand, the excess demand created by the benchmarked investors' hedging motive pushes up the price - similar to the symmetric information case. On the other hand, the substantial decline in price informativeness makes the payoff more uncertain, so that riskaverse investors command a lower price. In this setting, the second effect dominates, so that the equilibrium price declines. Interestingly, in the case of higher information costs $(\kappa=0.02)$, the price is actually $\mathrm{U}$-shaped in the size of the benchmarked institutions. That is, for lower $\Lambda$, the negative price effect resulting from a decline in price informativeness dominates, leading to a decline in price. In contrast, for larger fractions of benchmarked investors, the positive price effect induced by the hedging demand dominates so that the price starts to increase.

Not surprisingly, the stock market's return volatility is increasing in the share of benchmarked investors if one allows for endogenous information acquisition, as illustrated in Panel A of Figure $6 .{ }^{16}$ Particularly, as was discussed in the preceding section, an increase in the size of the benchmarked investors leads to a decline in price informativeness; that is, less of the variance of the payoff can be explained by the equilibrium price. Accordingly, the deviation between the time-2 price and the payoff revealed in period 3 increases, implying a more volatile stock market return.

\footnotetext{
${ }^{16}$ While the stock return volatility is slightly declining in our static economy with symmetric information, Basak and Pavlova (2013) show that return volatility is increasing in the presence of benchmarked investors if one considers a dynamic setting.
} 


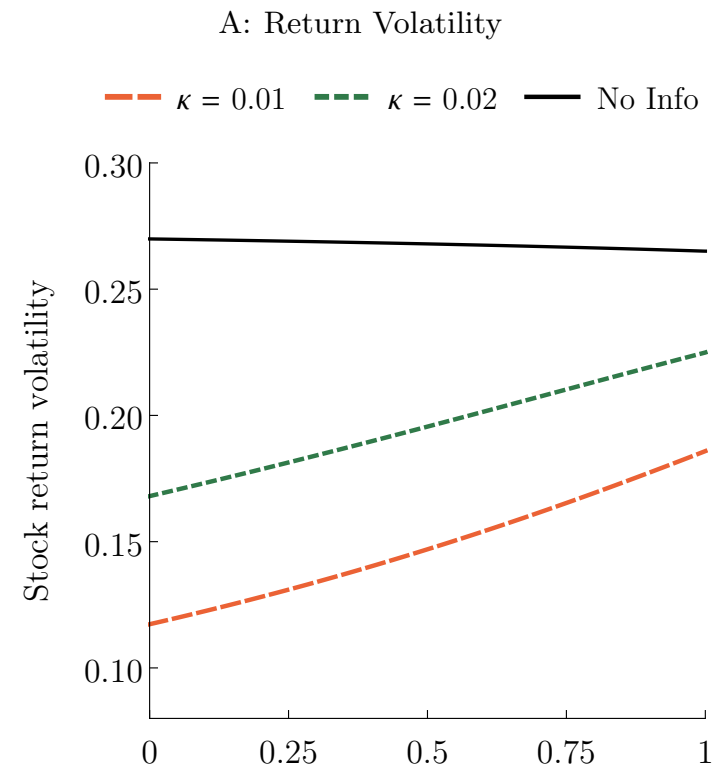

Fraction of benchmarked investors $(\Lambda)$
B: Expected Stock Return
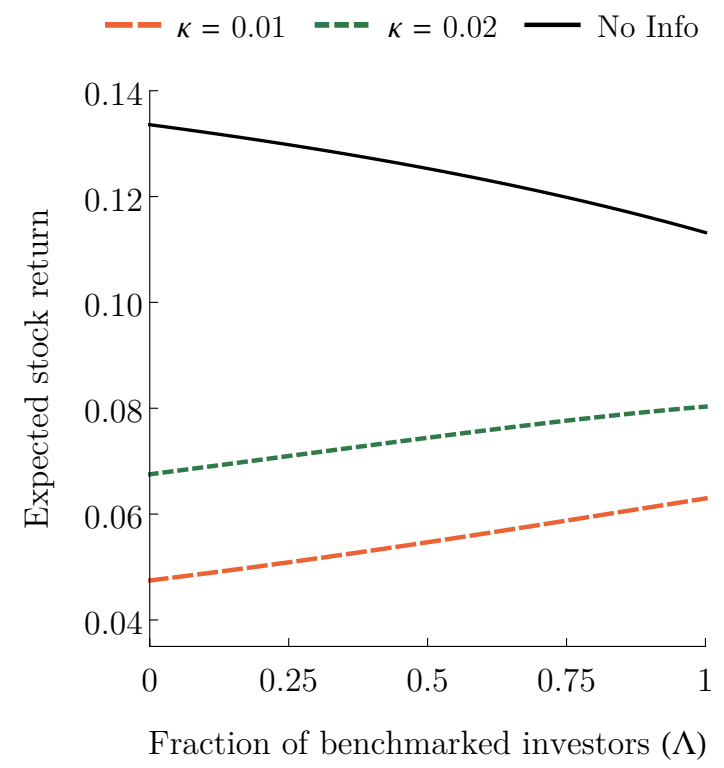

C: Sharpe Ratio

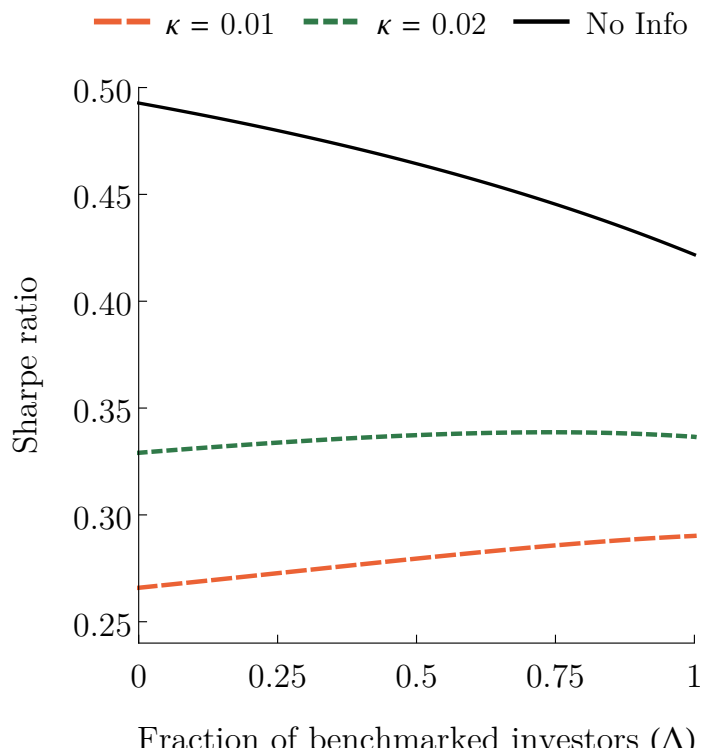

Figure 6: Moments of Stock Return. The figure shows the moments of the market return as a function of the share of benchmarked investors, $\Lambda$. Panels A to $\mathrm{C}$ show the expected conditional return volatility, the conditional expected return, and the expected conditional Sharpe ratio, respectively. The return moments are depicted for the case without information acquisition, i.e., with uninformed investors, as well as for the case with information acquisition, i.e., endogenous signals precision. Particularly, the return moments are shown for two levels of the information cost: $\kappa=0.01$ and $\kappa=0.02$. The results are based on the parameter values presented in Table 1. 
The fraction of benchmarked investors in the economy also affects the expected stock market return (Panel B of Figure 6). Particularly, in the case without information acquisition, the expected return is decreasing in the fraction of benchmarked investors, thereby generating a decline in the Sharpe ratio (Panel C). In contrast, with endogenous information choice, the expected return actually increases in the presence of benchmarked investors. This is the case if the negative price effect created by the lower level of price informativeness dominates. Moreover, for the cases with endogenous information acquisition, this increase leads also to an increase in the Sharpe ratio.

\subsection{Investors' Portfolio Returns}

The changes in the stock market's expected return and the investors' information choices also have an impact on the expected portfolio returns of the two groups of investors. Particularly, as Figure 7 shows, the abnormal portfolio return of the non-benchmarked investors, defined as an investor's expected return minus the market return, is increasing stronger than the abnormal return of benchmarked investors. That is, as a bigger share of wealth is managed by benchmarked investors, non-benchmarked investors can generate higher expected excess returns - especially so relative to their benchmarked peers.

Recall that the increase in the fraction of benchmarked investors leads to a decline in price informativeness, which strengthens the benefits of private information. Accordingly, the informational advantage of the non-benchmarked investors increases. This has two effects: First, non-benchmarked investors hold, on average, more of the stock market, which delivers a positive risk premium, because their superior information renders the investment less risky. Second, non-benchmarked investors' speculative activities are more profitable because their signals contain more information about the payoff. Finally, the increase in the expected return in the share of benchmarked investors amplifies the effect resulting from the, on average, higher portfolio share of the market for non-benchmarked investors. 


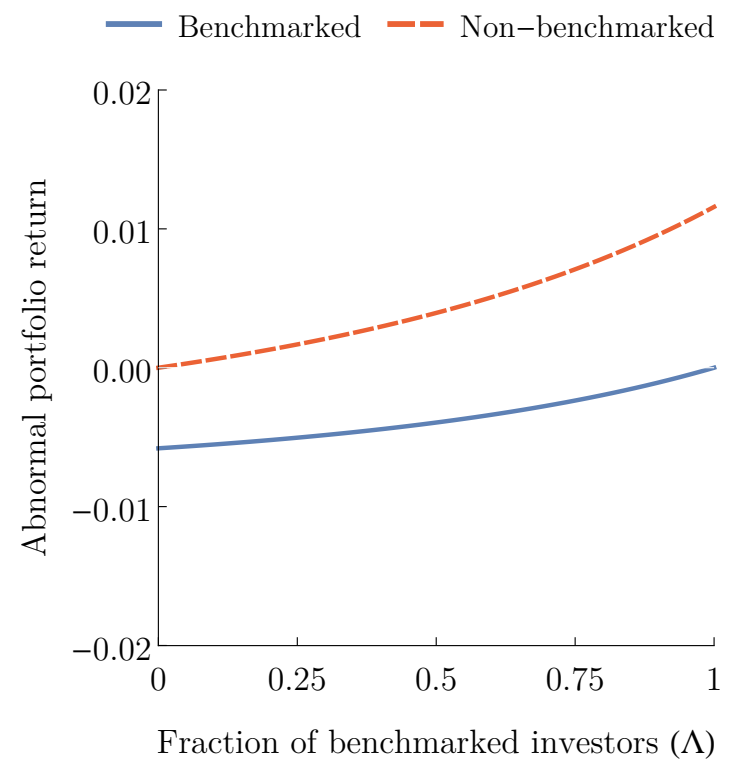

Figure 7: Abnormal Portfolio Returns. The figure shows the investors' expected abnormal portfolio returns, in excess of the average return of market, as a function of the share of benchmarked investors $\Lambda$. The results are based on the parameter values presented in Table 1.

\section{Equilibrium with Multiple Stocks}

Our results so far have been presented within the framework of a single-stock economy. Our objective in this section is to study how our results generalize in an economy with multiple stocks. Particularly, we study an economy with two risky stocks, with the first one also serving as the "index" (benchmark portfolio). We will refer to the first stock, $i=1$, as the "index stock" and the second stock, $i=2$, as the "non-index stock." Thus, the investor now strives to do well when the index stock does well.

\subsection{Portfolio and Information Choice}

Similar to the analysis of the single-stock economy, we first study a benchmarked investor's portfolio and information choice in partial equilibrium, that is, with exogenous stock prices, varying the degree of benchmarking in the investor's compensation scheme.

Specifically, conditional on an investor's posterior beliefs about the payoffs of the two stocks, he will choose the fraction of wealth to be invested into the stocks, $\phi_{j, i}, i \in\{1,2\}$, 
to maximize his expected utility (6), subject to the following budget equation:

$$
W_{j, 3}=W_{j, 2}\left(1+r_{f}+\phi_{j, 1} R_{1}^{e}+\phi_{j, 2} R_{2}^{e}\right),
$$

where $R_{i}^{e}$ denotes the excess return on stock $i$.

The investor's approximate portfolio shares are natural multi-stock generalizations of the single-stock case (9):

$$
\phi_{j, i} \approx\left(\frac{1}{\alpha}-\frac{\gamma_{j} \xi_{j}}{\alpha\left(1+\gamma_{j} \xi_{j}\right)}\right) \frac{E_{j}\left[R_{i}^{e} \mid \mathcal{F}_{j}\right]}{\operatorname{Var}_{j}\left[R_{i}^{e} \mid \mathcal{F}_{j}\right]}+\frac{\gamma_{j}}{\beta_{j}+\gamma_{j}} \frac{\operatorname{Cor}_{j}\left[R_{i}^{e}, R_{I} \mid \mathcal{F}_{j}\right] \sqrt{\operatorname{Var}_{j}\left[R_{i}^{e} \mid \mathcal{F}_{j}\right]}}{\sqrt{\operatorname{Var}_{j}\left[R_{I} \mid \mathcal{F}_{j}\right]}}
$$

Again, benchmarked investors hold the mean-variance portfolio of an investor with effective risk-aversion $\hat{\alpha}$, plus a hedging portfolio. Note that the hedging portfolio is now more refined. Particularly, the hedging demand for stock $i$ depends on the conditional correlation between a stock's return and the index return (the "quality" of the hedging instrument) as well as the ratio of the stock's and the index's conditional return volatilities (the "hedge ratio").

In our setting, in which the index is composed of one of the two stocks only, expression (14) can be further simplified. For the index stock, the correlation between the stock's return and the index's return as well as the ratio of their return volatilities is equal to one, so that the optimal portfolio share for the index stock simplifies to the one stock expression (10), with hedging demand $\frac{\gamma_{j}}{\beta_{j}+\gamma_{j}}$. In contrast, for the non-index stock, the hedging demand is practically zero, so that the optimal portfolio share is given solely by the standard mean-variance portfolio with effective risk-aversion $\hat{\alpha}$. Intuitively, because the two stocks' payoffs, signals, and noise are independent, their returns are also practically uncorrelated in equilibrium, which renders the correlation between the non-index stock and the index itself zero as well.

These effects are illustrated in Figure 8 for the case of an informed institutional investor, for various degrees of benchmarking $\gamma$. Specifically, Panel A depicts a pattern for the index 
A: Demand for Index Stock
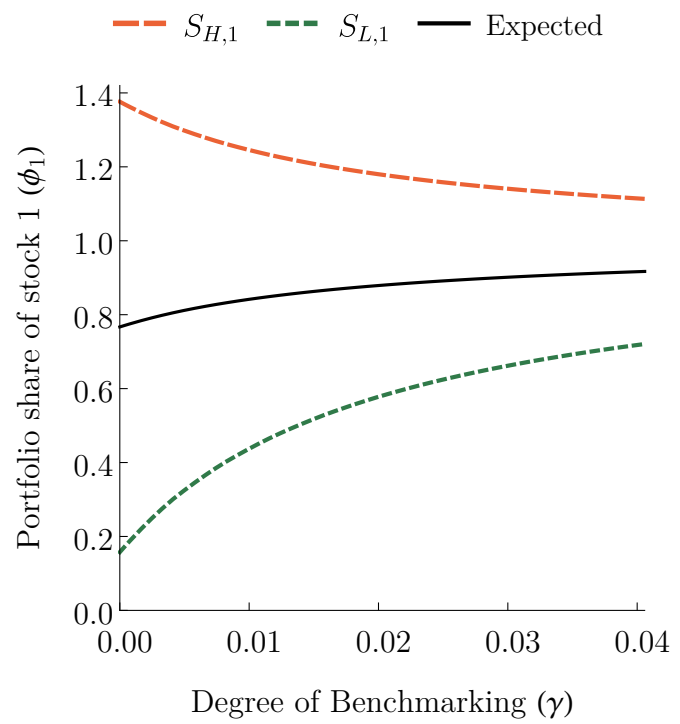

B: Demand for Non-Index Stock
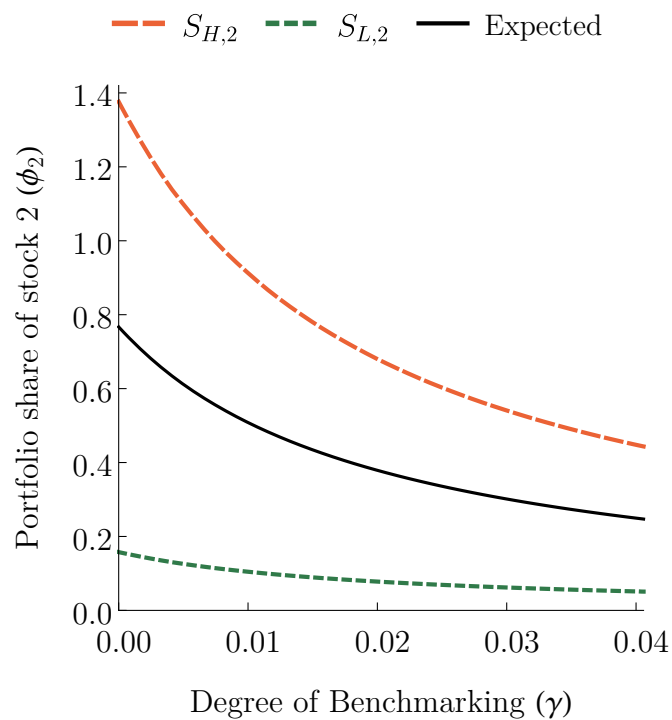

Figure 8: Stock Demand. The figure depicts the optimal period-2 portfolio choice of a single benchmarked investor, who takes the stock prices $P_{i}$ and the precision of his signals $x_{j, i}$ as given - as a function of the degree of benchmarking $\gamma$. The figure shows the expected portfolio share of stock $i$, as well as the optimal portfolio share conditional on the realization of the private signal $S_{j, i} \in\left\{S_{H}, S_{L}\right\}$. Panels A and B show the optimal portfolio share for the index $(i=1)$ and non-index stock $(i=2)$, respectively. The results are based on the parameter values presented in Table 1.

stock that is very similar to the one in the single-stock economy. The positive hedging demand more than offsets the drop in demand resulting from the increase in the investor's effective risk-aversion and leads, on average, to an increase in the share of the index stock in the investor's portfolio as the degree of benchmarking strengthens. At the same time, the increasing effective risk-aversion substantially reduces the investor's willingness to speculate based on private information, so that his portfolio becomes less sensitive to the realization of his private signal in the presence of benchmarking.

In contrast, Panel B shows that for the non-index stock, the expected holdings and the speculative demand decline in the degree of benchmarking. Because of the absence of a hedging demand, the increase in effective risk-aversion directly implies a lower expected portfolio share for the non-index stock. Moreover, the increase in effective risk-aversion reduces the speculative demand for the non-index stock - with the same magnitude as for the index stock. Thus, on aggregate, benchmarked investors tilt their portfolio toward index stocks. 
The investor's information choice problem is the same as for the single-stock case in (11), except that the investor now has to decide simultaneously on the signal precision for the two stocks, $x_{j, i}, i \in\{1,2\}$.

Unreported results ${ }^{17}$ show that an increase in the degree of benchmarking leads to a decline in signal precision for both stocks. Because the investor's speculative demand declines by the same magnitude for the two stocks - driven by the increase in his effective risk-aversion - and the hedging demand is information-insensitive, the investor's willingness to take large bets in the two stocks declines in parallel. Consequently, the sensitivity of the investor's portfolio and, in turn, the sensitivity of his period-1 expected utility with respect to private information about the two stocks declines by the same amount. Accordingly, the investor acquires exactly the same amount of information for the two stocks, that is, he chooses equal signal precisions in partial equilibrium.

\subsection{Price Informativeness in Equilibrium}

We now impose market clearing for the two stocks, as in (13), and, thus, turn to the equilibrium results with multiple stocks. Our key comparative statics parameter will be the fraction of benchmarked investors in the economy, $\Lambda$.

Similar to the case with a single risky asset, in equilibrium, financial markets aggregate the individual investors' private information through means of trading and market clearing. Consequently, the prices of the two stocks imperfectly reveal the private information acquired by the market participants.

Panel A of Figure 9 depicts price informativeness, measured as the fraction of the variance of the payoff of stock $i$, which can be explained by its stock price, $P_{i}$, alone. Price informativeness is declining for both stocks in the fraction of benchmarked institutional investors. This effect can be explained by the same mechanism as in the single-stock economy. An increase in the share of benchmarked investors implies that better informed, non-benchmarked investors are replaced by less informed benchmarked investors who trade

\footnotetext{
${ }^{17}$ Available upon request from the authors.
} 


\section{A: Price Informativeness}

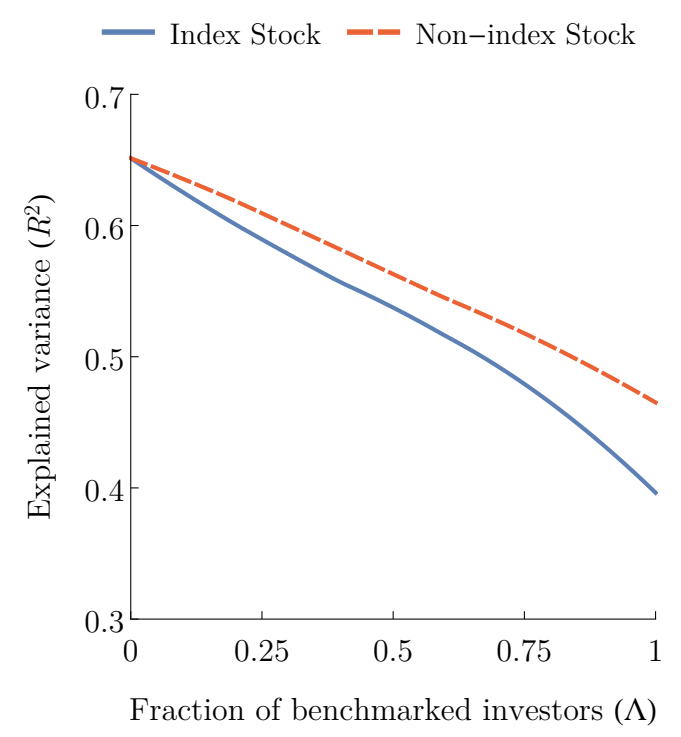

Figure 9: Informativeness of Stock Prices. This figure shows the fraction of the variance of the payoff $D_{i}$ that is explained by the stock prices $\left(R^{2}\right)$, as a function of the share of institutional investors $\Lambda$. The results are based on the parameter values presented in Table 1.

less aggressively, so that, on aggregate, less information is acquired and revealed through the stock prices.

Note that the decline in price informativeness is more pronounced for the index stock, which is due to the hedging demand of the benchmarked investors and CRRA-preferences. Effectively, the hedging demand, which is information-insensitive, reduces the supply of the index stock available for speculation. This, in turn, reduces the profitability of trading, so that investors trade less aggressively and, hence, less information is revealed through the price of the index stock relative to the non-index stock. Consequently, as the fraction of benchmarked investors increases, and so does their impact, the difference in price informativeness grows as well.

\subsection{Equilibrium Asset Prices and Returns}

These changes in the price informativeness of the two stocks have a direct impact on their equilibrium prices and returns, as is illustrated in Figure 10. 
Panel A shows that the prices of both stocks decline in the fraction of benchmarked investors in the economy, with the decline in the non-index stock's price being more pronounced. For the index stock, the effect is very much comparable to the price change in the single-stock economy and can be explained by the same two offsetting forces. On the one hand, the hedging demand of the benchmarked investors creates an excess demand for the index stock. Because the stock is in fixed supply, for markets to clear, the price would have to go up. On the other hand, the decrease in the index stock's price informativeness renders investments into the stock riskier, so that risk-averse investors command a lower price. On aggregate, the second effect dominates, leading to the slight decline in the expected price of the index stock. For the non-index stock there is no hedging demand that would (partially) offset the decline in price resulting from lower price informativeness, so that the price declines more. As the impact of the benchmarked investors, and with it, the aggregate hedging demand, increases, the difference between the expected prices of the two stocks widens.

Panel B of Figure 10 shows that the expected conditional Sharpe ratio is higher for the non-index stock, which can be explained through "equilibrium incentives." Due to the hedging demand of the benchmarked investors, in order to ensure market clearing, nonbenchmarked investors must be induced to tilt their portfolio toward the non-index stock, which is achieved through a higher Sharpe ratio.

Panels $\mathrm{C}$ and D illustrate that, for both stocks, the expected conditional return and the expected conditional volatility are increasing, as a bigger share of wealth is managed by benchmarked investors. The increase in the expected return for the non-index stock is stronger, due to the more pronounced decline in its expected price, and the gap in expected returns widens with the share of benchmarked investors. The decline in both stock's price informativeness explains the increase in the stocks' return volatilities, with a more pronounced increase of the index stock's return volatility due to the stronger decline in price informativeness. The correlation between the index and the non-index stock (unreported) is basically not affected. 
A: Stock Price

— Index Stock -- Non-index Stock

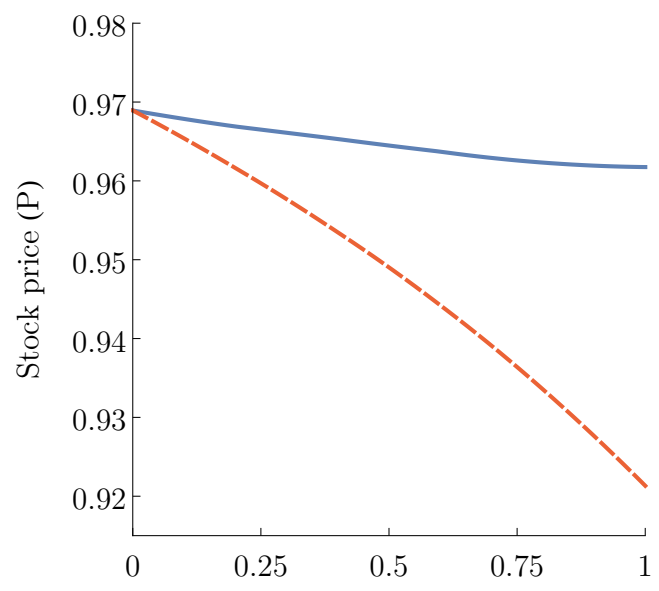

Fraction of benchmarked investors $(\Lambda)$

C: Expected Stock Return

— Index Stock $\quad-$ Non-index Stock

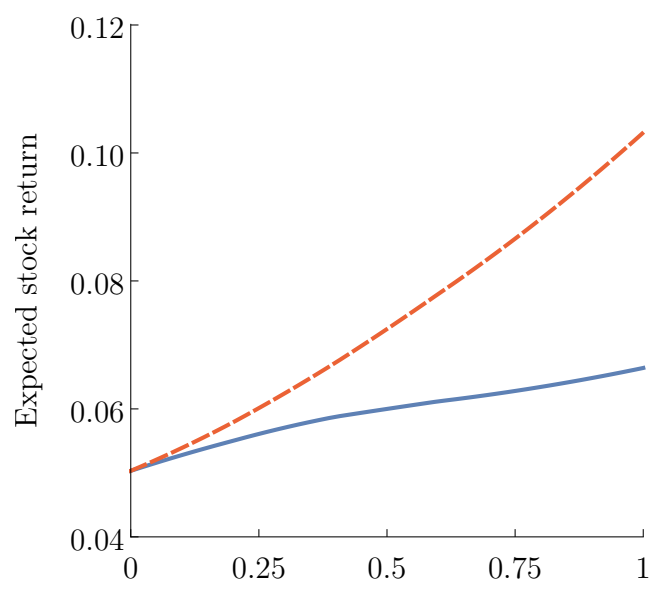

Fraction of benchmarked investors $(\Lambda)$
B: Sharpe Ratio

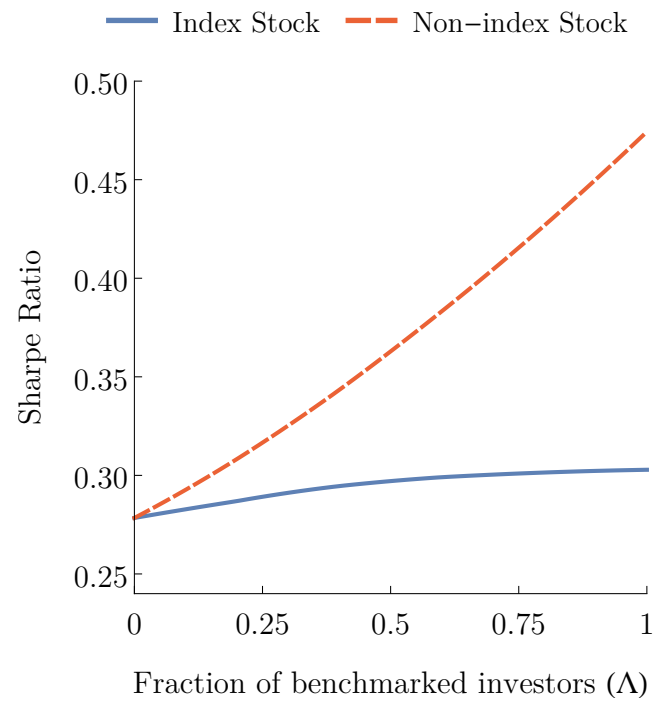

D: Return Volatility

— Index Stock - - Non-index Stock

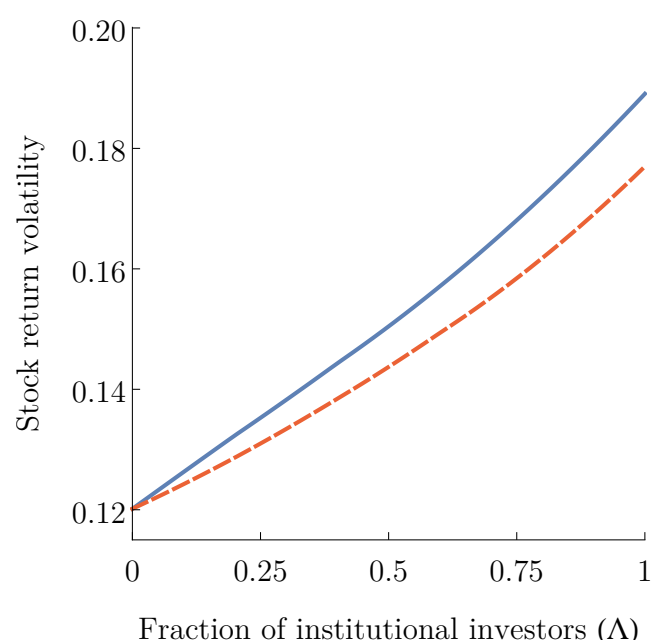

Figure 10: Stock Price and Moments of Stock Returns. The figure shows the expected prices of the two stocks and their return moments as a function of the share of benchmarked investors, $\Lambda$. Panels A to D show the expected stock prices, the expected conditional Sharpe ratios, the conditional expected returns, and the expected conditional return volatilities, respectively. The results are based on the parameter values presented in Table 1.

Note that these changes in the individual stocks' prices and returns also have direct implications for the stock market as a whole. Particularly, the decline in both stocks' equilibrium prices implies a decline in the value of the aggregate market as well. Similarly, because the return correlation is virtually unchanged, the increase in both stocks' return 
volatilities implies a substantial increase in the volatility of the market return. Despite this increase in the return volatility of the market, its Sharpe ratio is increasing in the fraction of benchmarked investors. This can be attributed to the decline in aggregate, effective risk-aversion, which means that, in order for markets to clear, investors must be induced to hold more of the market.

\section{Summary of Key Predictions and Empirical Implications}

Relative performance concerns play a key role in the decisions of many institutional investors who are in the business of acquiring information and using that information for portfolio management. In this paper, we develop an economic framework that explicitly accounts for benchmarking and the joint determination of investors' portfolio and information choice in equilibrium.

We highlight a novel economic channel through which benchmarking affects the portfolio allocation and information choice of institutional investors: Relative performance concerns lead to an increase in the effective risk-aversion of a benchmarked institutional investor, particular so as his "surplus performance" relative to the index declines. Intuitively, this increase in risk-aversion reduces the investor's willingness to speculate and, consequently, his desire to acquire information, with important implications for prices and returns.

Some of our predictions, particularly those comparing index and non-index stocks, are similar to findings in the asset pricing literature and supported by the empirical literature on "index effects." For example, similar to Cuoco and Kaniel (2011) and Basak and Pavlova (2013), we find that the bias of benchmarked investors' portfolios in favor of index stocks results in higher prices and return volatilities, but lower Sharpe ratios for index stocks. But, analyzing portfolio and information choices jointly also delivers new insights that are unique to our economic framework. For instance, our analysis suggests that benchmarking reduces price informativeness. As a direct consequence, the prices of all risky assets and the market portfolio might decline. The decline in price informativeness also leads to a substantial increase in return volatilities in the presence of benchmarked investors - in 
contrast to the results in Cuoco and Kaniel (2011), in which information choice is absent and, thus, price informativeness is unchanged. Finally, with less informative prices, private information gains importance, leading to a substantial out-performance of better informed non-benchmarked investors.

While there is a growing body of literature on the asset pricing implications of institutional investors, our paper helps to better understand their joint information and portfolio choice. However, many aspects of institutional investors' impact on financial markets are not well understood yet. For example, extensions of our framework could be used to understand the optimal size of benchmarked investors in the economy (Pástor and Stambaugh (2012)) or the "implicit costs" of passive investing (French (2008)). 


\section{Appendix}

\section{A Proofs}

\section{Proof of Lemma 1}

Rewrite the investors' compensation $C_{j}$ from equation (2) as

$$
C_{j}\left(W_{j, 3}, R_{I}\right)=\beta_{j} W_{j, 3}+\gamma_{j}\left(W_{j, 3}-\left(1+R_{I}\right)\right)=\left(\beta_{j}+\gamma_{j}\right) W_{j, 3}-\gamma_{j}\left(1+R_{I}\right),
$$

plug it into utility function (3), and take the derivatives with respect to wealth $W_{j, 3}$ :

$$
\frac{\partial U_{j}\left(C_{j}\right)}{\partial W_{j, 3}}=C_{j}^{-\alpha}\left(\beta_{j}+\gamma_{j}\right) ; \quad \frac{\partial^{2} U_{j}\left(C_{j}\right)}{\partial W_{j, 3}^{2}}=(-\alpha) C_{j}^{-(\alpha+1)}\left(\beta_{j}+\gamma_{j}\right)^{2} .
$$

Thus, the local curvature of the utility function, $\hat{\alpha}_{j}$, is given by

$$
\begin{aligned}
\hat{\alpha}_{j} & \equiv-\frac{W_{j, 3} \partial^{2} U_{j} / \partial W_{j, 3}^{2}}{\partial U_{j} / \partial W_{j, 3}}=\frac{-W_{j, 3}(-\alpha) C_{j}^{-(\alpha+1)}\left(\beta_{j}+\gamma_{j}\right)^{2}}{C_{j}^{-\alpha}\left(\beta_{j}+\gamma_{j}\right)} \\
& =\alpha\left(\frac{1}{\beta_{j}+\gamma_{j}} \frac{\left(\beta_{j}+\gamma_{j}\right) W_{j, 3}-\gamma_{j}\left(1+R_{I}\right)}{W_{j, 3}}\right)^{-1} \\
& =\alpha\left(\frac{1}{\beta_{j}+\gamma_{j}}\left(\left(\beta_{j}+\gamma_{j}\right)-\gamma_{j} \frac{\left(1+R_{I}\right)}{1+R_{j, F}}\right)\right)^{-1}=\alpha\left(1-\frac{\gamma_{j}}{\beta_{j}+\gamma_{j}}\left(\frac{1+R_{j, F}}{1+R_{I}}\right)^{-1}\right)^{-1} .
\end{aligned}
$$

Assume that $\gamma_{j}>0, \beta_{j}>0$ and $R_{I}>-1$. Then we get

$$
\hat{\alpha}_{j}=\alpha(1-\underbrace{\frac{\gamma_{j}}{\beta_{j}+\gamma_{j}}}_{>0} \underbrace{\left(\frac{1+R_{j, F}}{1+R_{I}}\right)^{-1}}_{>0})^{-1}>\alpha .
$$


Taking the derivative of $\hat{\alpha}_{j}$ with respect to $\gamma_{j}$ and $\left(1+R_{j, F}\right) /\left(1+R_{I}\right)$ yields

$$
\begin{gathered}
\frac{\partial \hat{\alpha}_{j}}{\partial \gamma_{j}}=\alpha\left(1-\frac{\gamma_{j}}{\beta_{j}+\gamma_{j}}\left(\frac{1+R_{j, F}}{1+R_{I}}\right)^{-1}\right)^{-2}(-1)\left(-\left(\frac{1+R_{j, F}}{1+R_{I}}\right)^{-1}\left(\frac{1}{\beta_{j}+\gamma_{j}}+\frac{-\gamma_{j}}{\left(\beta_{j}+\gamma_{j}\right)^{2}}\right)\right) \\
=\alpha \underbrace{\left(1-\frac{\gamma_{j}}{\beta_{j}+\gamma_{j}}\left(\frac{1+R_{j, F}}{1+R_{I}}\right)^{-1}\right)^{-2}}_{>0} \underbrace{\left(\frac{1+R_{j, F}}{1+R_{I}}\right)^{-1}}_{>0} \underbrace{\frac{\beta_{j}}{\left(\beta_{j}+\gamma_{j}\right)^{2}}>0}_{>0} \\
\frac{\partial \hat{\alpha}_{j}}{\partial \frac{1+R_{j, F}}{1+R_{I}}}=\alpha\left(1-\frac{\gamma_{j}}{\beta_{j}+\gamma_{j}}\left(\frac{1+R_{j, F}}{1+R_{I}}\right)^{-1}\right)^{-2}(-1) \frac{-\gamma_{j}}{\beta_{j}+\gamma_{j}}(-1)\left(\frac{1+R_{j, F}}{1+R_{I}}\right)^{-2} \\
=\underbrace{-\alpha}_{<0} \underbrace{\left(1-\frac{\gamma_{j}}{\beta_{j}+\gamma_{j}}\left(\frac{1+R_{j, F}}{1+R_{I}}\right)^{-1}\right)^{-2}}_{>0} \underbrace{\frac{\gamma_{j}}{\beta_{j}+\gamma_{j}}}_{>0} \underbrace{\left(\frac{1+R_{j, F}}{1+R_{I}}\right)^{-2}}_{>0}<0 .
\end{gathered}
$$

\section{Derivation of Portfolio Share Approximation for the Single-Stock Economy}

For ease of exposition, define the normalized compensation, $\hat{C}_{j}$, as $\hat{C}_{j}=\frac{C_{j}}{\beta_{j}+\gamma_{j}}$. This compensation can be easily rewritten as

$$
\begin{aligned}
\hat{C}_{j}\left(W_{j, 3}, R_{I}\right) & =\frac{1}{\beta_{j}+\gamma_{j}}\left(\left(\beta_{j}+\gamma_{j}\right) W_{j, 3}-\gamma_{j}\left(1+R_{I}\right)\right) \\
& =\left(1+r_{f}+\phi_{j} R^{e}\right)-\frac{\gamma_{j}}{\beta_{j}+\gamma_{j}}\left(1+R_{I}\right),
\end{aligned}
$$

where we replaced $W_{j, 3}$ with the wealth dynamics in (7), and, without loss of generality, normalized $W_{j, 2}$ to 1 .

Now, consider the case in which the excess return on the stock market, $R^{e}$, and the return on the index, $R_{I}$, are jointly normally distributed, with expectations $\mu_{S}$ and $\mu_{I}$, variances $\sigma_{S}^{2}$ and $\sigma_{I}^{2}$ and covariance $\sigma_{S, I}$. Then the normalized compensation $\hat{C}_{j}$ is normally distributed with the following mean and variance

$$
\begin{gathered}
E\left[\hat{C}_{j}\right]=\left(1+r_{f}+\phi_{j} \mu_{S}\right)-\frac{\gamma_{j}}{\beta_{j}+\gamma_{j}}\left(1+\mu_{I}\right), \\
\operatorname{Var}\left(\hat{C}_{j}\right)=\phi_{j}^{2} \sigma_{S}^{2}+\frac{\gamma_{j}^{2}}{\left(\beta_{j}+\gamma_{j}\right)^{2}} \sigma_{I}^{2}+2 \phi_{j} \frac{\gamma_{j}}{\beta_{j}+\gamma_{j}} \sigma_{S, I} .
\end{gathered}
$$


In the region where the mean, $E\left[\hat{C}_{j}\right]$ is close to one, the normally distributed variable $\hat{C}_{j}$ can be closely approximated using a log-normally distributed variable with the same mean and variance: ${ }^{18}$

$$
\hat{C}_{j} \approx \exp \left(-1+E\left[\hat{C}_{j}\right]-\frac{1}{2} \operatorname{Var}\left(\hat{C}_{j}\right)+\sqrt{\operatorname{Var}\left(\hat{C}_{j}\right)} \nu\right)
$$

where $\nu$ denotes a standard normal distributed variable.

Optimizing utility (3) over $\hat{C}_{j}$ is equivalent to maximizing the utility over $C_{j}$ because the term $\frac{1}{\beta_{j}+\gamma_{j}}$ is simply a multiplicative constant. Accordingly, we can write the portfolio optimization problem as:

$$
\max _{\phi_{j}} E\left[\frac{\hat{C}_{j}^{1-\alpha}}{1-\alpha}\right]
$$

which can, by computing the expectation for approximation (A1), be simplified to

$$
\max _{\phi_{j}} \exp \left((1-\alpha)\left(-1+E\left[\hat{C}_{j}\right]-\frac{1}{2} \operatorname{Var}\left(\hat{C}_{j}\right)\right)+\frac{1}{2}(1-\alpha)^{2} \operatorname{Var}\left(\hat{C}_{j}\right)\right) .
$$

The first-order condition with respect to $\phi_{j}$ yields

$$
\mu_{S}-\phi_{j} \sigma_{S}^{2} \alpha+\frac{\gamma_{j}}{\beta_{j}+\gamma_{j}} \sigma_{S, I} \alpha=0
$$

such that

$$
\begin{aligned}
\phi_{j} & =\frac{1}{\alpha} \frac{\mu_{S}}{\sigma_{S}^{2}}+\frac{\gamma_{j}}{\beta_{j}+\gamma_{j}} \frac{\sigma_{S, I}}{\sigma_{S}^{2}} \\
& =\frac{1}{\alpha} \frac{\mu_{S}}{\sigma_{S}^{2}}+\frac{\gamma_{j}}{\beta_{j}+\gamma_{j}} \operatorname{Cor}\left(R^{e}, R_{I}\right) \frac{\sigma_{I}}{\sigma_{S}} .
\end{aligned}
$$

\footnotetext{
${ }^{18}$ See also van Nieuwerburgh and Veldkamp (2010).
} 
Note, however, that due to the approximation, the increase in risk-aversion is "lost." Particularly, one can rewrite the approximation for $\hat{C}_{j}$ in (A1) as

$$
\begin{aligned}
& \exp \left(r+\phi_{j} \mu_{S}-\frac{1}{2} \phi_{j}^{2} \sigma_{S}^{2}+\phi_{j} \sigma_{S} \nu\right) \\
& \quad \times \exp \left(-\frac{\gamma_{j}}{\beta_{j}+\gamma_{j}}\left(1+\mu_{I}\right)-\frac{1}{2}\left(\frac{\gamma_{j}^{2} \sigma_{I}^{2}}{\left(\beta_{j}+\gamma_{j}\right)^{2}}-2 \phi_{j} \frac{\gamma_{j}}{\beta_{j}+\gamma_{j}} \sigma_{S, I}\right)-\frac{\gamma_{j}}{\beta_{j}+\gamma_{j}} \sigma_{I} \nu\right),
\end{aligned}
$$

where the first component is the log-normal approximation of wealth $W_{j, 3}$. Hence, the normalized compensation $\hat{C}_{j}$ can also be approximately written as

$$
\hat{C}_{j} \approx W_{3, j} \times \exp \left(-\frac{\gamma_{j}}{\beta_{j}+\gamma_{j}}\left(1+\mu_{I}\right)-\frac{1}{2}\left(\frac{\gamma_{j}^{2} \sigma_{I}^{2}}{\left(\beta_{j}+\gamma_{j}\right)^{2}}-2 \phi_{j} \frac{\gamma_{j}}{\beta_{j}+\gamma_{j}} \sigma_{S, I}\right)-\frac{\gamma_{j}}{\beta_{j}+\gamma_{j}} \sigma_{I} \nu\right)
$$

which makes it apparent that it is linear in wealth $W_{3, j}$.

Accordingly, by taking the first and second derivative of the utility function (3) over the approximated normalized compensation, as in (A3), with respect to wealth $W_{j, 3}$, one arrives at a local curvature of the approximated utility function equal to $\alpha$. That is, the approximation does not take into account the change in risk-aversion resulting from benchmarking.

Intuitively, one can simply take this change in risk-aversion into account by replacing $\alpha$ in the approximated portfolio share (A2) with $\hat{\alpha}$, as in (4). Recognizing that, in the single-stock economy, the correlation between the market return and the index return as well as the ratio of their return volatilities is one, yields equation (10), from which equation (9) follows immediately.

Figure 11 illustrates the precision of the approximation for the portfolio choice problem of the informed benchmarked investor discussed in Section 2.1. The approximated portfolio shares (dashed lines) closely resemble the true portfolio shares (solid lines) for both signal realizations.

\section{B Numerical Algorithm}

Due to our deviation from the CARA-normal framework, the equilibrium price function in our economy is nonlinear, and its specific functional form is unknown. Accordingly, we 


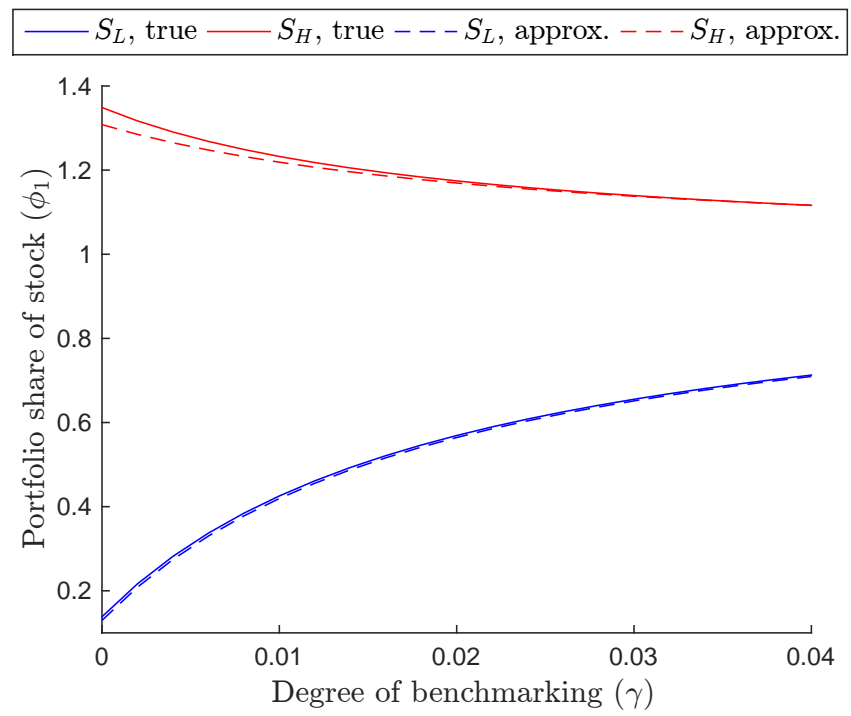

Figure 11: Conditional Stock Demand. The figure shows the optimal portfolio share of the stock market for an informed institutional investors with benchmarking degree $\gamma$. The demand is shown conditional on a high or low signal realization. Moreover, the figure shows the true as well as the approximated demand (based on (9)). The results are based on the same parameters as in Section 2.1 and illustrated in Panel C of Figure 2.

have to rely on a numerical algorithm to solve for the equilibrium. In the following, we provide some details of the algorithm that we have developed. For ease of exposition, we concentrate on the single-stock economy.

Particularly, as described in Section 3.1, in equilibrium, each investor must have rational expectations, as specified in (5), and each investor must choose a portfolio to maximize his expected utility (6), subject to his period-2 budget equation, and the stock market needs to clear for all realizations of the underlying (unobservable) payoff $D$ and the (unobservable) noisy supply $z$. In addition, in period 1 , each investor must choose a signal precision to maximizes his expected utility (6), subject to his period-1 budget equation.

We discretize the state space for the noisy supply $z$, using $N$ grid points. The full equation system then consists of the following set of equations: First, $2 \times 2 \times 2 \times N$ "posterior equations" (5), describing the posterior beliefs for the two groups of investors, the two (current) underlying payoff realizations, the two (future) possible realizations of the payoff, and the $N$ grid points of the supply; second, $2 \times 2 \times N$ portfolio first-order conditions (8), again for the two groups of investors, the two underlying payoff realizations and the $N$ grid points of the supply; third, $2 \times N$ market clearing condition (13) for the two underlying payoff realizations, and the $N$ grid points of the supply; and fourth, two information acquisition 
first-order conditions (12) for the two groups of investors. That is, in total, we arrive at $14 N+2$ equations.

The unknowns of the equation system are given by the following variables: First, $2 \times$ $2 \times 2 \times N$ posterior beliefs $\pi_{\cdot, k}$ for the two groups of investors, the two (current) underlying payoff realizations, the two (future) possible realizations of the payoff, and the $N$ grid points of the supply; second, $2 \times 2 \times N$ portfolio shares of the stock $\phi_{j}$ for the two groups of investors, ${ }^{19}$ the two underlying payoff realizations, and the $N$ grid points of the supply; third, $2 \times N$ stock prices $P$ for the two underlying payoff realizations and the $N$ grid points of the supply; and fourth, 2 signal precisions $x_{j}$ for the two groups of investors, which makes in total $14 N+2$ variables.

The equation system cannot be solved recursively because the period- 1 choice of the signal precision $x_{j}$ affects the period-2 posterior beliefs and, in turn, the portfolio choices and market clearing. Accordingly, we solve this large fixed-point problem globally using Mathematica.

\footnotetext{
${ }^{19}$ The investment into the bond is simply given by wealth minus the investment into the stock and the information cost.
} 


\section{References}

Admati, A. R., and P. Pfleiderer, 1997, "Does It All Add Up? Benchmarks and the Compensation of Active Portfolio Managers," Journal of Business, 70(3), 323-350.

Albagli, E., C. Hellwig, and A. Tsyvinski, 2014, "Dynamic Dispersed Information and the Credit Spread Puzzle," NBER Working Paper No. 19788.

Bakke, T.-E., and T. M. Whited, 2010, "Which Firms Follow the Market? An Analysis of Corporate Investment Decisions," Review of Financial Studies, 23(5), 1941-1980.

Barlevy, G., and P. Veronesi, 2000, "Information Acquisition in Financial Markets," Review of Economic Studies, 67(1), 79-90.

Basak, S., and A. Pavlova, 2013, "Asset Prices and Institutional Investors," American Economic Review, 103(5), 1728-1758.

— , 2016, "A Model of Financialization of Commodities," The Journal of Finance, 71(4), 1511-1556.

Bernardo, A. E., and K. L. Judd, 2000, "Asset Market Equilibrium with General Tastes, Returns, and Informational Asymmetries," Journal of Financial Markets, 3(1), 17-43.

Bond, P., A. Edmans, and I. Goldstein, 2012, "The Real Effects of Financial Markets," Annual Review of Financial Economics, 4(1), 339-360.

Bond, P., and D. García, 2016, "The Equilibrium Consequences of Indexing," Working Paper.

Brennan, M., 1993, "Agency and Asset Pricing," Unpublished Manuscript.

Breon-Drish, B., 2015, "On Existence and Uniqueness of Equilibrium in a Class of Noisy Rational Expectations Models," The Review of Economic Studies, 82(3), 868-921.

Breugem, M., 2016, "On the Dispersion of Skil and Size in Active Management: Multi-Agent Dynamic Equilibrium with Endogenous Information," Working Paper.

Buffa, A., and I. Hodor, 2017, "Institutional Investors, Heterogeneous Benchmarks and the Comovement of Asset Prices," Working Paper.

Buffa, A., D. Vayanos, and P. Woolley, 2014, "Asset Management Contracts and Equilibrium Prices," CEPR Discussion Papers.

Campbell, J., and J. Cochrane, 1999, "By Force of Habit: A Consumption-Based Explanation of Aggregate Stock Market Behavior," Journal of Political Economy, 107(2), $205-251$.

Chabakauri, G., K. Yuan, and K. E. Zachariadis, 2016, "Multi-Asset Noisy Rational Expectations Equilibrium with Contingent Claims," Working paper.

Chen, Q., I. Goldstein, and W. Jiang, 2007, "Price Informativeness and Investment Sensitivity to Stock Price," Review of Financial Studies, 20(3), 619-650.

Cuoco, D., and R. Kaniel, 2011, "Equilibrium Prices in the Presence of Delegated Portfolio Management," Journal of Financial Economics, 101(2), 264-296. 
Edmans, A., I. Goldstein, and W. Jiang, 2012, "The Real Effects of Financial Markets: The Impact of Prices on Takeovers," The Journal of Finance, 67(3), 933-971.

Farboodi, M., and L. Veldkamp, 2016, "The Long-Run Evolution of the Financial Sector," Working Paper.

Foucault, T., and L. Frésard, 2012, "Cross-Listing, Investment Sensitivity to Stock Price, and the Learning Hypothesis," Review of Financial Studies, 25(11), 3305-3350.

French, K. R., 2008, "Presidential Address: The Cost of Active Investing," The Journal of Finance, 63(4), 1537-1573.

García, D., and G. Strobl, 2011, "Relative Wealth Concerns and Complementarities in Information Acquisition," The Review of Financial Studies, 24(1), 169.

García, D., and J. M. Vanden, 2009, "Information Acquisition and Mutual Funds," Journal of Economic Theory, 144(5), 1965-1995.

Griffin, J. M., J. H. Harris, and S. Topaloglu, 2003, "The Dynamics of Institutional and Individual Trading," The Journal of Finance, 58(6), 2285-2320.

Grossman, S., 1976, "On the Efficiency of Competitive Stock Markets where Trades have diverse Information," The Journal of Finance, 31(2), 573-585.

Grossman, S. J., and J. E. Stiglitz, 1980, "On the Impossibility of Informationally Efficient Markets," American Economic Review, 70(3), 393-408.

Hellwig, M. F., 1980, "On the Aggregation of Information in Competitive Markets," Journal of Economic Theory, 22(3), 477-498.

Ibert, M., R. Kaniel, S. van Nieuwerburgh, and R. Vestman, 2017, "Are Mutual Fund Managers Paid for Investment Skill?," Working Paper.

Kacperczyk, M., S. van Nieuwerburgh, and L. Veldkamp, 2016, "A Rational Theory of Mutual Funds' Attention Allocation," Econometrica, 84(2), 571-626.

Malamud, S., and E. Petrov, 2014, "Portfolio Delegation and Market Efficiency," Working Paper.

Milgrom, P., and N. Stokey, 1982, "Information, Trade and Common Knowledge," Journal of Economic Theory, 26(1), 17-27.

Pástor, L., and R. F. Stambaugh, 2012, "On the Size of the Active Management Industry," Journal of Political Economy, 120(4), 740-781.

Peress, J., 2003, "Wealth, Information Acquisition, and Portfolio Choice," Review of Financial Studies, 17(3), 879-914.

Qiu, Z., 2012, "An Institutional REE Model with Relative Performance," Working Paper.

Sotes-Paladino, J., and F. Zapatero, 2016, "A Rationale for Benchmarking in Money Management," Working Paper.

Stambaugh, R. F., 2014, "Presidential Address: Investment Noise and Trends," The Journal of Finance, 69(4), 1415-1453. 
U.S. Securities and Exchange Commission, 2013, "Institutional Investors: Power and Responsibility," Technical Report.

van Nieuwerburgh, S., and L. Veldkamp, 2010, "Information Acquisition and UnderDiversification," Review of Economic Studies, 77(2), 779-805.

Verrecchia, R. E., 1982, "Information Acquisition in a Noisy Rational Expectations Economy," Econometrica, 50(6), 1415-1430. 